\title{
LDU FACTORIZATION RESULTS FOR BI-INFINITE AND SEMI-INFINITE SCALAR AND BLOCK TOEPLITZ MATRICES
}

\author{
C. van der Mee, G. Rodriguez, S. Seatzu (1)
}

ABSTRACT - In this article various existence results for the $L D U$-factorization of semiinfinite and bi-infinite scalar and block Toeplitz matrices and numerical methods for computing them are reviewed. Moreover, their application to the orthonormalization of splines is indicated. Both banded and non-banded Toeplitz matrices are considered. Extensive use is made of matrix polynomial theory. Results on the approximation by the $L D U$-factorizations of finite sections are discussed. The generalization of the results to the $L D U$-factorization of multi-index Toeplitz matrices is outlined.

\section{Introduction}

Let $A$ be a bi-infinite Toeplitz matrix $A=\left(A_{i-j}\right)_{i, j \in \mathbb{Z}}$ where $\mathbb{Z}$ is the set of integers and $A_{h}, h \in \mathbb{Z}$, are $k \times k$ matrices, so that $A_{h}$ is a scalar if $k=1$ and a $k \times k$ matrix if $k>1$.

We consider the factorization

$$
\text { (1.1) } A=L D U \text {, }
$$

where $L=\left(L_{i-j}\right)_{i, j \in \mathbb{Z}}$ is a lower triangular matrix, $U=\left(U_{i-j}\right)_{i, j \in \mathbb{Z}}$ is an upper triangular matrix and $D=\left(D_{i-j}\right)_{i, j \in \mathbb{Z}}$ is a diagonal matrix. Thus, $A_{h}, L_{h}, U_{h}$ and $D_{h}, h \in \mathbb{Z}$, are scalars if $k=1$ and $k \times k$ matrices if $k>1$.

This factorization, which exists under very general hypotheses, is not unique. We are especially interested in obtaining efficient methods for finding the unique factorization such that $D$ is nonsingular and $L$ and $U$ are boundedly invertible on the Hilbert space $l_{2}(\mathbb{Z})$ of all square summable bi-infinite complex sequences with $L_{0}=U_{0}=I$ ( $I$ being 1 if $k=1$ and the identity matrix of order $k$ if

- Received: november 16, 1996

(1) Department of Mathematics, University of Cagliari. Viale Merello 92, 09123 Cagliari, Italy. Email: cornelis@vaxca2.unica.it, rodriguez@unica.it, seatzu@unica.it. Partially supported by the Italian Ministry of University and Scientific and Technological Research and by the Italian National Research Council 
$k>1)$ and further $L^{-1}$ and $U^{-1}$ are lower and upper triangular Toeplitz matrices, respectively.

The outline of the paper is as follows: in $\S 2$ we give a review of several results on the $L D U$ factorization of bi-infinite and semi-infinite scalar and block Toeplitz matrices; in $\S 3$ we synthetically illustrate the algorithms we are aware of to obtain the spectral factorization of bi-infinite real banded and positive definite scalar Toeplitz matrices; in $\S 4$ we review some useful properties of matrix polynomials; in $\S 5$ we prove the necessary and sufficient conditions for the existence of the $L D U$ factorization of bi-infinite block-tridiagonal Toeplitz matrices. The proof is constructive and leads to a numerical method for obtaining the factorization that we are looking for. In $\S 6$ and $\S 7$ we illustrate two comparatively new research lines and their relevance to the solution of some problems typical of approximation theory. Finally, in $\S 8$ we generalize the $L D U$-factorization results to multi-index Toeplitz and non-Toeplitz matrices.

\section{Factorization of bi-infinite Toeplitz matrices}

Let $l_{2}(\mathbb{Z})$ be the Hilbert space of all square summable bi-infinite sequences with norm

$$
\|x\|^{2}=\sum_{j \in \mathbb{Z}}\left|x_{j}\right|^{2}, \quad x=\left(x_{j}\right)_{j \in \mathbb{Z}},
$$

and inner product

$$
\langle x, y\rangle=x^{*} y=\sum_{j \in \mathbb{Z}} \vec{x}_{j} y_{j}, \quad x=\left(x_{j}\right)_{j \in \mathbb{Z},}, \quad y=\left(y_{j}\right)_{j \in \mathbb{Z}},
$$

where $x^{*}=\left(\bar{x}_{j}\right)_{j \in \mathbb{Z}}$.

Consider the bi-infinite Toeplitz matrix $A=\left(A_{i-j}\right)_{i, j \in \mathbb{Z}}$, defined as any bounded linear operator $A$ on $l_{2}(\mathbb{Z})$ that commutes with the right shift $S\left(x_{n}\right)_{n \in \mathbb{Z}}=\left(x_{n-1}\right)_{n \in \mathbb{Z}}$. For Toeplitz matrices in the Wiener class we have

$$
\sum_{j=-\infty}^{\infty}\left|A_{j}\right|<+\infty
$$

Define the symbol by

$$
\hat{A}(z)=\sum_{j=-\infty}^{\infty} A_{j} z^{j}, \quad|z|=1
$$

Then $L D U$-factorization of $A$ with factors and their inverses in the Wiener class amounts to the Wiener-Hopf factorization

(2.1) $\hat{A}(z)=\hat{L}(z) D \hat{U}(z), \quad|z|=1$,

where $D$ is a nonzero constant, $\hat{L}(z)$ and $\hat{L}(z)^{-1}$ are continuous in $|z| \leq 1$ and analytic in $|z|<1$, and $\hat{U}(z)$ and $\hat{U}(z)^{-1}$ are continuous in $|z| \geq 1$ and analytic 
in $|z|>1$ (including $\infty$ ). The functions $\hat{L}(z)$ and $\hat{U}(z)$ and the constant $D$ are connected to the factors $L, U$ and $D$ as follows

$$
\hat{L}(z)=\sum_{j=0}^{\infty} L_{j} z^{j}, \quad \hat{U}(z)=\sum_{j=0}^{\infty} U_{-j} z^{-j} \quad \text { and } \quad D=D_{0} .
$$

For $\hat{A}(z)$ in the Wiener class, a Wiener-Hopf factorization (2.1) exists (and its factors $\hat{L}(z), \hat{U}(z)$ and their reciprocals are sums of absolutely convergent Fourier series) if and only if $\hat{A}(z) \neq 0$ for $|z|=1$ and has zero winding number with respect to the origin $[20,14]$. Here the winding number is the unique $\kappa \in \mathbb{Z}$ such that $\log \left(z^{-\kappa} \hat{A}(z)\right)$ is continuous in $|z|=1$.

Let $A^{T}$. denote the transpose of $A$ and $A^{*}$ the adjoint of $A$, i.e. $\left(A^{*}\right)_{i j}=(\bar{A})_{j i}$. A self-adjoint matrix is called positive definite whenever there is a constant $\kappa>0$ such that

$$
x^{*} A x \geq \kappa x^{*} x, \quad x \in l_{2}(\mathbb{Z}) .
$$

Clearly, a Toeplitz matrix $A$ is positive definite if and only if $\hat{A}(z)$ is strictly positive on the unit circle $(\exists \varepsilon>0: \hat{A}(z) \geq \varepsilon,|z|=1)$. Moreover, a positive definite Toeplitz matrix $A$ in the Wiener class has an $L D U$-factorization with factors in the Wiener class. Said otherwise, (2.1) exists if $\hat{A}(z)$ is the sum of an absolutely convergent Fourier series and is strictly positive on the unit circle $[20,14]$. In this case,

$$
D>0, \quad \hat{U}(z)=\hat{L}\left(\bar{z}^{-1}\right)^{*},
$$

and therefore

$$
\hat{A}(z)=\tilde{L}(z) \tilde{L}\left(\bar{z}^{-1}\right)^{*}, \quad \tilde{L}(z)=\hat{L}(z) D^{1 / 2},
$$

leads to a Cholesky factorization of $A$.

For $A$ in the Wiener class with factorization (1.1), the finite truncations

$$
A^{(n)}=\left(A_{i-j}\right)_{i, j=-n}^{n}
$$

have an $L D U$-factorization for sufficiently large $n$ and the $L D U$-factors in $A^{(n)}$ converge strongly to those of $A$ (cf. [14]).

Now take positive weights $w_{j}$ such that $w_{i+j} \leq w_{i} w_{j}$, and consider the biinfinite Toeplitz matrix $A$ in the weighted Wiener class:

$$
\sum_{j=-\infty}^{\infty} w_{j}\left|A_{j}\right|<+\infty
$$

Putting $\rho_{ \pm}=\lim _{j \rightarrow \pm \infty}\left(w_{j}\right)^{1 / j}$, so that $0<\rho_{-} \leq \rho_{+}<+\infty$, we find that $A$ has a bounded inverse in the same weighted Wiener class if and only if $\hat{A}(z) \neq 0$ when $\rho_{-} \leq|z| \leq \rho_{+}$(cf. [9]). If $\hat{A}(z)$ also has zero winding number with respect to the origin, the $L D U$-factors of $A$ and their inverses are in the above weighted Wiener class.

The following special cases are of particular interest: 
(a) $w_{j}=r^{|j|}$ for some $r \geq 1$. Then $\rho_{-}=1 / r$ and $\rho_{+}=r$.

(b) $w_{j}=(1+|j|)^{\alpha}$ for some $\alpha \geq 0$. Then $\rho_{ \pm}=1$.

If $A$ is banded (i.e. $A_{j}=0$ for $|j|>m$ ), then $z^{m} \hat{A}(z)$ is a polynomial of degree $2 m$. Moreover, if $0<\rho_{-} \leq 1 \leq \rho_{+}<+\infty, \hat{A}(z) \neq 0$ for $\rho_{-} \leq|z| \leq \rho_{+}$and $\hat{A}(z)$ has zero winding number with respect to the origin, then the $L D U$-factors of $A$ and their inverses are in the weighted Wiener class with weights $w_{j}=\rho_{+}^{j}$ and $w_{-j}=\rho_{-}^{j}$ for $j \geq 0$.

If $A$ is positive definite and banded, the Cholesky factors of $A$ are banded and their inverses satisfy

$$
\sum_{j=1}^{\infty} \rho_{+}^{j}\left|\left[\hat{L}^{-1}\right]_{j}\right|<+\infty, \quad \sum_{j=1}^{\infty} \frac{1}{\rho_{-}^{j}}\left|\left[\hat{U}^{-1}\right]_{-j}\right|<+\infty
$$

if $\hat{A}(z) \neq 0$ for $\rho_{-} \leq|z| \leq \rho_{+}$for certain $\rho_{ \pm}$with $0<\rho_{-}<1<\rho_{+}<+\infty$. The inequalities (2.2) are also true if $A$ satisfies

$$
\sum_{j=0}^{\infty} \rho_{+}^{j}\left|A_{j}\right|<+\infty, \quad \sum_{j=0}^{\infty} \frac{1}{\rho_{-}^{j}}\left|A_{-j}\right|<+\infty
$$

for certain $0<\rho_{-} \leq 1 \leq \rho_{+}<+\infty$, and $\hat{A}(z) \neq 0$ for $\rho_{-} \leq|z| \leq \rho_{+}$and $\hat{A}(z)$ has zero winding number with respect to the origin. In particular, if $A_{j}$ and $A_{-j}$ are exponentially decreasing (i.e., if $A_{ \pm j}=O\left(s^{ \pm j}\right)$ as $j \rightarrow+\infty$ for certain $s_{ \pm} \in(0,1)$ ), (2.3) is true for any $\rho_{+} \in\left(1,1 / s_{+}\right)$and $\rho_{-}>\left(1 / s_{-}\right)$.

For bi-infinite $k \times k$ block Toeplitz matrices $A$, one defines the Wiener class as the class of matrices $A$ for which

$$
\sum_{j=-\infty}^{\infty}\left\|A_{j}\right\|<+\infty
$$

Its symbol

$$
\hat{A}(z)=\sum_{j=-\infty}^{\infty} z^{j} A_{j}, \quad|z|=1,
$$

is a $k \times k$ matrix function. Then $L D U$-factorization of $A$ with factors and its inverses in the Wiener class amounts to the Wiener-Hopf factorization

$$
\hat{A}(z)=\hat{L}(z) D \hat{U}(z), \quad|z|=1,
$$

where $D$ is a constant invertible matrix, $\hat{L}(z)$ and $\hat{L}(z)^{-1}$ are continuous in $|z| \leq 1$ and analytic in $|z|<1$, and $\hat{U}(z)$ and $\hat{U}(z)^{-1}$ are continuous in $|z| \geq 1$ and analytic in $|z|>1$ (including $\infty$ ). In that case we have the necessary condition for the existence of (2.1)

$$
\operatorname{det} \hat{A}(z)=\operatorname{det} \hat{L}(z) \operatorname{det} D \operatorname{det} \hat{U}(z), \quad|z|=1 .
$$


Necessary and sufficient conditions can be given in terms of the so-called partial indices of $\hat{A}(z)[14,9]$, but except in some very specific cases (such as positive definite $\hat{A}(z)$, rational matrix functions, and matrix polynomials) these indices cannot be easily computed.

If $A$ is a positive definite $k \times k$ block Toeplitz matrix in the Wiener class, there exists a Wiener-Hopf factorization as in (2.1); in that case $D$ is positive definite and $\hat{U}(z)=\hat{L}\left(\bar{z}^{-1}\right)^{*}$. Moreover, $A$ has a Cholesky factorization $A=\mathrm{LL}^{*}$ where

$$
\hat{\mathrm{E}}(z)=\hat{L}(z) D^{1 / 2}=\hat{U}\left(\bar{z}^{-1}\right)^{*} D^{1 / 2} .
$$

\section{Real symmetric and banded scalar matrices}

For its relevance in the applications, we now outline the five methods we are aware of for the spectral factorization of banded and symmetric real matrices. This problem is crucial, e.g., to the identification of the asymptotic behavior of the Gram-Schmidt iteration for the orthonormalization of a large number of integer translates of a fixed function $[17,18]$ and also in techniques used for wavelet construction $[7,27]$. This section is largely adapted from [19].

Let $A=\left(a_{i-j}\right)_{i, j \in \mathbb{Z}}$ be a real symmetric and banded Toeplitz matrix and let

$$
a(z)=\sum_{j=-m}^{m} a_{j} z^{j}
$$

be its symbol, that is a Laurent polynomial of degree $m$ such that $a_{j}=\vec{a}_{j}=a_{-j}$, $j=1,2, \ldots, m$. We denote the space of all such functions by $\mathcal{S}_{m}$. When $a \in \mathcal{S}_{m}$ has the property that

(3.1) $a(z)>0, \quad|z|=1$,

the problem we consider is to find real numbers $\gamma_{0}, \gamma_{1}, \ldots, \gamma_{m}$ such that

$$
\gamma(z)=\sum_{j=0}^{m} \gamma_{j} z^{j}
$$

has all of its roots outside the unit disk and such that

(3.2) $\gamma(z) \gamma\left(z^{-1}\right)=a(z), \quad z \in \mathbb{C} \backslash\{0\}$.

To guarantee the uniqueness of the factorization we impose the normalization $\gamma(0)>0$ on the polynomial $\gamma(z)$. The existence of such a factorization is wellknown and attributed to Fejér [23, pg. 117].

The first method we wish to outline is due to F. Bauer $[2,3]$. As the Laurent polynomial a satisfies (3.1) the matrix $A$ is positive definite on $l_{2}(\mathbb{Z})$. Moreover, the factorization (3.2) corresponds to the factorization

$$
A=\Gamma \Gamma^{T}
$$


where $\Gamma=\left(\gamma_{i-j}\right)_{i, j \in \mathbb{Z}}$ is the banded lower triangular matrix formed by the coefficients of $\gamma(z)$.

Let $A_{+}$be the semi-infinite compression of the bi-infinite matrix $A$ given by

$$
A_{+}=\left(A_{i j}\right)_{i, j \in \mathbb{Z}_{+}}, \quad \mathbb{Z}_{+}=\{0,1,2, \ldots\},
$$

and $A_{n}, n \in \mathbb{Z}_{+}$, be the sequence of finite compressions of $A$ defined by

$$
A_{n}=\left(A_{i j}\right)_{i, j=0,1, \ldots, n} \text {. }
$$

As a result, each finite compression $A_{n}$ has a unique Cholesky factorization

$$
L_{n} L_{n}^{T}=A_{n}
$$

where $L_{n}$ is a lower triangular matrix with positive diagonal elements. As we increase $n$ to $n+1$ the matrix $A_{n+1}$ agrees with $A_{n}$ in its first $n+1$ rows and columns. Likewise $L_{n+1}$ has its first $n+1$ rows and columns equal to those of $L_{n}$. Therefore we may consider $L_{n}$ to be the $n$-th finite section of a semi-infinite matrix $L$ which is the unique Cholesky factor of $A_{+}$.

Bauer proved that $\left(L_{n}\right)_{i j} \rightarrow \gamma_{i-j}$ as $n \rightarrow \infty$, and in [17] it has been proved that the elements of the semi-infinite Cholesky factor $L$ approach those of $\Gamma$ exponentially, that is, there exist $c>0$ and $\rho \in(0,1)$ such that for all $i, j \in \mathbb{Z}_{+}$

(3.3) $\left|l_{i j}-\gamma_{i-j}\right| \leq c \rho^{i}$,

so that the Bauer method represents an iterative linearly convergent method.

A second iterative method, which is quadratically convergent, is due to Wilson [28], cf. [19]. His idea is to write equation (3.2) as the following equivalent system of quadratic equations:

$$
\sum_{j=0}^{m-i} \gamma_{j} \gamma_{j+i}=a_{i}, \quad i=0,1, \ldots, m
$$

His approach consists of the use of a Newton-Raphson method for solving system (3.4). Wilson proved that, if $a(z)$ has no zeros on the unit circle, for a suitable and easily made choice of the starting values $\gamma_{0}^{(0)}, \gamma_{1}^{(0)}, \ldots, \gamma_{m}^{(0)}$, the iteration is selfcorrecting and converges quadratically to the required solution. For an effective implementation of this as well as of the next method we refer to [19].

The third algorithm we mention is based on the computation of the zeros of the Laurent polynomial $a(z)$. In order to improve the effectiveness of the method, in view of the symmetry of its coefficients, we take $w=z+z^{-1}$ and consider the polynomial

$$
c(w):=a(z(w))=\sum_{j=0}^{m} c_{j} w^{j}
$$


whose coefficients $c_{0}, c_{1}, \ldots, c_{m}$ can be expressed recursively in terms of the original coefficients $a_{0}, a_{1}, \ldots, a_{m}$.

The zeros $w_{i}, i=1,2, \ldots, m$, of the polynomial $c(w)$ can then be evaluated by computing the eigenvalues of its companion matrix by a QR method [16]. Next, recalling that $w=z+z^{-1}$, we obtain the $2 m$ roots $z_{i}$ of $a(z)$, which we order by decreasing modulus.

As the coefficients of $a(z)$ are real and symmetric, if $z_{i}$ is a root so are $\bar{z}_{i}$ and $z_{i}^{-1}$, so that if $z_{i}$ is outside the unit circle, $z_{i}^{-1}$ is inside and conversely.

The polynomial $\gamma(z)$ that we are looking for can then be constructed by taking all the roots $z_{j}$ (including $\tilde{z}_{j}$ ) outside the unit circle.

The fourth method which has been tested in [19] is the minimum phase factorization. It is based on the following observation.

Let $a(z)$ have no zeros on the unit circle. For any $g \in \Pi_{m}$ normalized so that $g(0)=1$ we consider the minimization problem

$$
\min _{g \in \Pi_{m}, g(0)=1} \int_{-\pi}^{\pi}\left|g\left(e^{i \theta}\right)\right|^{2} \frac{d \theta}{a\left(e^{i \theta}\right)} .
$$

The solution of this minimization problem is given by

$$
g_{\mathrm{opt}}(z):=\frac{\gamma(z)}{\gamma(0)}
$$

where $\gamma$ is the polynomial in (3.2) we seek. The coefficients of $g_{\text {opt }}$ are determined by solving a Toeplitz linear system.

The final method we review is very popular in signal processing and goes by the name of the cepstral algorithm $[4,22]$. It is based on some results on the factorization of an absolute convergent Fourier series on the unit circle, discovered independently by M.G. Krein [20] and by A. Calderón, F. Spitzer and H. Widom [5]. Let us recall the basic idea of this method.

As the Laurent polynomial $a(z)$ is strictly positive on the unit circle, by the Wiener-Lévy theorem concerning trigonometric series, the function $\log a(z)$ can be written as an absolutely convergent Laurent series

$$
b(z):=\log a(z)=\sum_{j \in \mathbb{Z}} b_{j} z^{j} .
$$

Moreover, the coefficients $b_{j}$, as those of $a(z)$, are even in $j$ and decay exponentially, since $\log a(z)$ has an extension as an analytic function in some annulus $r^{-1}<|z|<r, r>1$, containing the unit circle.

Splitting the Laurent series in the form

$$
b(z)=b_{-}(z)+b_{+}(z)
$$

where

$$
b_{ \pm}(z):=\sum_{j=0}^{\infty}\left(1-\frac{1}{2} \delta_{j}\right) b_{ \pm j} z^{ \pm j}, \quad\left|z^{ \pm}\right| \leq 1
$$


we obtain the factorization (3.2) of the required type, where

$$
\gamma(z):=\exp \left(b_{+}(z)\right)
$$

is analytic and zero free in the disk $\{z:|z| \leq r\}$ and normalized so that $\gamma(0)>0$, and $\delta_{j}=0$ for $j \neq 0$ and $\delta_{0}=1$. Note that $\gamma$ above is indeed a polynomial of degree at most $m$. A MATLAB implementation of this method has been proposed in [1].

In [19] it was found that there is a huge disparity between the methods, and that all of them except for the Wilson method are significantly affected by the variation in magnitude of the coefficients of the Laurent polynomial, by the closeness of the zeros of this polynomial to the unit circle and by their spacing.

To explain how the spectral factorization of Laurent polynomials which are positive on the unit circle has been used to identify the limiting profile of splines obtained by orthonormalizing a large finite set of B-splines with integer knots, we recall some results from [17].

Let $B_{k}$ be the $(k+1)$-fold convolution of the characteristic function of the interval $[0,1)$ (Schoenberg [26, pg. 11] called $B_{k}$ the $(k+1)$-st order forward $B$-spline). Consider the integer translates of $B_{k}$, that is

$$
\phi_{i}:=B_{k}(\cdot-i), \quad i \in \mathbb{Z} \text {. }
$$

Suppose $n$ is an integer such that $n \geq k+1$ and take $m=n-k-1$. Next we orthonormalize on $[0, n]$ the functions $\phi_{0}, \ldots, \phi_{m}$ recursively by a Gram Schmidt process. In this way we generate the functions $\psi_{0}^{n}, \ldots, \psi_{m}^{n}$ having the property that

$$
\int_{0}^{n} \psi_{i}^{n}(x) \psi_{j}^{n}(x) d x=\delta_{i j}, \quad i, j=0,1, \ldots, m
$$

The asymptotic behavior of this recursion as $n \rightarrow \infty$ has been studied in [17]. Among other things the authors proved that there exist a function $O(x), x \in \mathbb{R}$, and constants $c>0, \mu \in(0,1)$ such that for any $n$ and $0 \leq i \leq m$

$$
\left|\psi_{i}^{n}(x)-O_{i}(x)\right| \leq c \mu^{i}, \quad x \in \mathbb{R}_{4},
$$

where $O_{i}(x):=O(x-i)$. This function $O$ has the property that

$$
\int_{\mathbb{R}} O(x) O_{j}(x) d x=\delta_{j 0}, \quad j \in \mathbb{Z},
$$

and for some constants $\left\{\gamma_{j}\right\}_{j \in \mathbb{Z}_{+}}$which decay exponentially

$$
O=\sum_{j \in \mathbb{Z}_{+}} \gamma_{j} \phi_{-j}
$$

The constants $\left\{\gamma_{j}\right\}_{j \in \mathbb{Z}_{+}}$can be identified in the following manner. We consider the Toeplitz matrix

$$
T=\left(t_{i-j}\right)_{i, j \in \mathbb{Z}}
$$


where

$$
t_{i}:=\int_{\mathbb{R}} B_{k}(t) B_{k}(t-i) d t=B_{2 k+1}(k+1+i), \quad i \in \mathbb{Z},
$$

and let $\pi_{n}(z), z \in \mathbb{C}$, stand for the Euler-Frobenius polynomial of degree $n-1$ [26, pg. 22]. Then

$$
t(z):=\sum_{j \in \mathbb{Z}} t_{j} z^{j}=\frac{1}{(2 k+1) !} z^{-k} \pi_{2 k+1}(z), \quad z \in \mathbb{C} .
$$

Moreover, from [26, pg. 22], we know that $\pi_{2 k+1}(z)$ has $2 k$ simple negative zeros $\lambda_{1}<\cdots<\lambda_{2 k}<0$ such that $\lambda_{1} \lambda_{2 k}=\cdots=\lambda_{k} \lambda_{k+1}=1$. Consequently, we can write $t(z)$ in the form

$$
t(z)=h(z) h\left(z^{-1}\right), \quad z \in \mathbb{C} \backslash\{0\},
$$

where $h(z)$ is a real polynomial of degree $m$ having its zeros outside the unit circle at $\lambda_{1}, \ldots, \lambda_{k}$ with $h(0)>0$. Since $h(z)$ has no zeros in the unit disk we can expand its reciprocal in some neighborhood of the unit disk as

$$
\gamma(z):=\frac{1}{h(z)}=\sum_{j \in \mathbb{Z}_{+}} \gamma_{j} z^{j}
$$

where the constants $\left\{\gamma_{j}\right\}_{j \in \mathbb{Z}_{+}}$necessarily decay exponentially. These constants determine the limiting profile by formula (3.5) (Figure 1).
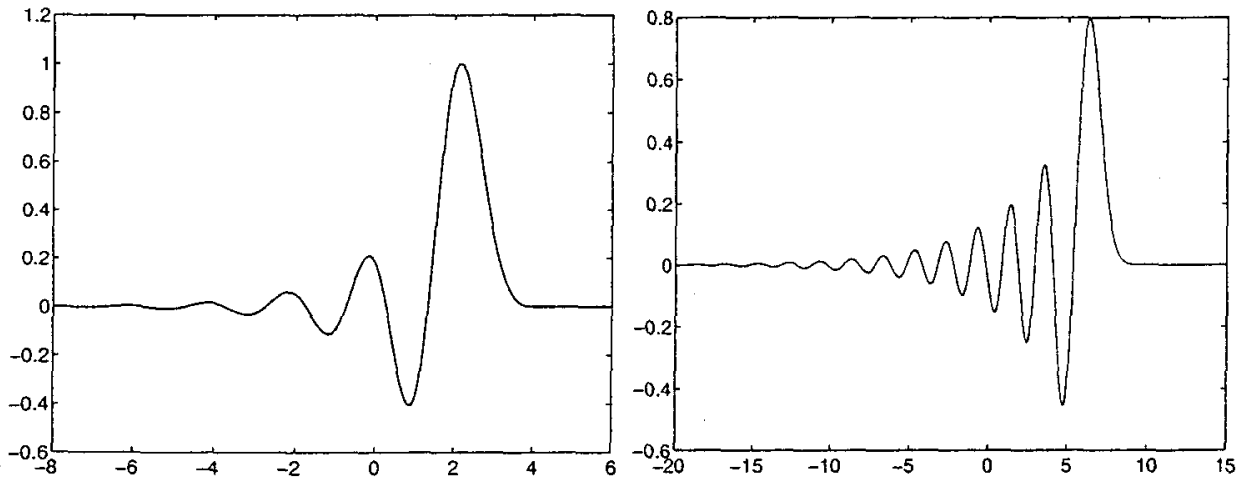

Figure 1: 0 -splines of degree 3 and 10

\section{Review of properties of matrix polynomials}

Jordan chains of matrix polynomials. Let $W(z)$ be a $k \times k$ matrix polynomial, i.e., a polynomial in $z$ whose coefficients are real or complex $k \times k$ matrices. Then det $W(z)$ either vanishes identically or is a scalar polynomial in $z$. We call $z_{0} \in \mathbb{C}$ an eigenvalue of $W$ if $\operatorname{det} W\left(z_{0}\right)=0$, and $\varphi_{0} \in \mathbb{C}^{k}$ an eigenvector 
of $W$ corresponding to the eigenvalue $z_{0}$ if $\varphi_{0} \neq 0$ and $W\left(z_{0}\right) \varphi_{0}=0$. The set of eigenvalues of $W$ is called the spectrum of $W$; it either coincides with the entire complex plane (if $\operatorname{det} W(z) \equiv 0$ ) or with the finite set of zeros of $\operatorname{det} W(z)$.

Let $z_{0}$ be an eigenvalue of $W$. Then the $m$-uple of vectors $\varphi_{0}, \varphi_{1}, \ldots, \varphi_{m-1}$ in $\mathbb{C}^{k}$ is called a Jordan chain at $z_{0}$ of length $m$ if $\varphi_{0} \neq 0$ and the upper triangular linear system

$$
\sum_{j=0}^{k} \frac{W^{(j)}\left(z_{0}\right)}{j !} \phi_{k-j}=0, \quad k=0,1, \ldots, m-1
$$

is satisfied. If one considers the subspace of $\mathbb{C}^{k}$ spanned by the vectors constituting the Jordan chains at the eigenvalue $z_{0}$ and orders the Jordan chains, say, according to decreasing length until one has a basis of this subspace, the lengths of these chains are the so-called partial multiplicities of $W$ at the eigenvalue $z_{0}$. They do not depend on the specific choice of the Jordan chains but only on $W$. The sum of the partial multiplicities at the eigenvalue $z_{0}$ coincides with the order of $z_{0}$ as a zero of $\operatorname{det} W(z)$ and is called the algebraic multiplicity of $z_{0}$; the dimension of the kernel of $W\left(z_{0}\right)$, which coincides with the number of Jordan chains at $z_{0}$, is called its geometric multiplicity. This definition appears in [25] in the context of operator polynomials.

In matrix algebra one usually considers the situation $W(z)=z-T$ where $T$ is a $k \times k$ matrix and the leading coefficient is the identity matrix. The partial multiplicities are then exactly the sizes of the Jordan blocks at the eigenvalue $z_{0}$ of $T$.

A definition of partial multiplicity through root vectors for meromorphic operator functions, clearly equivalent to the present one in the case of matrix polynomials, appears in [13].

Spectral pairs of matrix polynomials. Let us introduce some basic facts about matrix polynomials $[11,12,25]$. Consider the monic matrix polynomial

$$
\mathcal{P}(z)=z^{l}+z^{l-1} A_{l-1}+\cdots+z A_{1}+A_{0}
$$

where the coefficients are $k \times k$ matrices; here monic means that the leading coefficient is the identity matrix. Since $\operatorname{det} \mathcal{P}(z)$ is a scalar polynomial of degree $k l$, there are exactly $k l$ eigenvalues, when counted according to algebraic multiplicity. Let $z_{1}, \ldots, z_{p}$ be the distinct eigenvalues of $\mathcal{P}(z)$. For each $j$, we construct a pair of matrices $X_{j}$ (of size $k \times m_{j}$ where $m_{j}$ is the multiplicity of $z_{j}$ as a zero of $\operatorname{det} \mathcal{P}(z)$ ) and $T_{j}$ (of size $m_{j} \times m_{j}$ ) as follows:

$$
\begin{aligned}
& X_{j}=\left[\begin{array}{llllllllll}
x_{11}^{(j)} & \cdots & x_{1 r_{1}}^{(j)} & x_{21}^{(j)} & \cdots & x_{2 r_{2}}^{(j)} & \cdots & x_{q 1}^{(j)} & \cdots & x_{q r_{q}}^{(j)}
\end{array}\right] \\
& T_{j}=\operatorname{diag}\left(J_{r_{1}}\left(z_{j}\right), J_{r_{2}}\left(z_{j}\right), \ldots, J_{r_{q}}\left(z_{j}\right)\right) \text {. }
\end{aligned}
$$

Here $x_{s 1}^{(j)}, x_{s 2}^{(j)}, \ldots, x_{s r_{s}}^{(j)}$ for $s=1, \ldots, q$ are the Jordan chains for $\mathcal{P}(z)$ corresponding to $z_{j}$ such that the eigenvectors $x_{11}^{(j)}, x_{21}^{(j)}, \ldots, x_{q 1}^{(j)}$ are linearly independent and 
$r_{1}+r_{2}+\cdots+r_{q}=m_{j}$, and $J_{r_{s}}\left(z_{j}\right)$ is the $r_{s} \times r_{s}$ upper triangular Jordan block with eigenvalue $z_{j}$. Finally, put

$$
X=\left[\begin{array}{llll}
X_{1} & X_{2} & \cdots & X_{p}
\end{array}\right], \quad T=\operatorname{diag}\left(T_{1}, T_{2}, \ldots, T_{p}\right)
$$

One can show $[11,12]$ that the $k l \times k l$ matrix

(4.4) $\operatorname{col}\left[X T^{j}\right]_{j=0}^{l-1}:=\left[\begin{array}{c}X \\ X T \\ \vdots \\ X T^{l-1}\end{array}\right]$

is invertible and that

(4.5) $X T^{l}+\sum_{j=0}^{l-1} A_{j} X T^{j}=0$.

More generally, let $\mathcal{P}(z)$ be the monic $k \times k$ matrix polynomial given by (4.2). Then the pair of matrices $(X, T)$, where $X$ is of size $k \times k l$ and $T$ is of size $k l \times k l$, is called a right spectral pair for the polynomial $\mathcal{P}(z)$ if the operator in (4.4) is invertible and (4.5) holds. Thus the pair $(X, T)$ given by (4.3) is a right spectral pair for $\mathcal{P}(z)$. According to [25], Theorem 2.1.1, for every couple of right spectral pairs $\left(X_{1}, T_{1}\right)$ and $\left(X_{2}, T_{2}\right)$ of the same monic matrix polynomial there exists a unique invertible matrix $S$ such that

$$
X_{1}=X_{2} S, \quad T_{1}=S^{-1} T_{2} S .
$$

Analogously, $(T, Y)$, where $Y$ is of size $k l \times k$ and $T$ is of size $k l \times k l$, is called a left spectral pair if the matrix

$$
\left[\begin{array}{llll}
Y & T Y & \cdots & T^{l-l} Y
\end{array}\right]
$$

is invertible and

$$
T^{l} Y+\sum_{j=0}^{l-1} T^{j} Y A_{j}=0 .
$$

One can prove that for every couple of left spectral pairs $\left(T_{1}, Y_{1}\right)$ and $\left(T_{2}, Y_{2}\right)$ of the same monic matrix polynomial there exists a unique invertible matrix $S$ such that

$$
Y_{1}=S^{-1} Y_{2}, \quad T_{1}=S^{-1} T_{2} S .
$$

According to [25], Theorem 2.2.1, we have the following: If $\left(X, T_{1}\right)$ is a right spectral pair and $\left(T_{2}, Y\right)$ is a left spectral pair of the same monic matrix polynomial $\mathcal{P}(z)$ given by $(4.2)$, then

$$
\mathcal{P}(z)=z^{l}-X T_{1}^{l}\left(V_{1}+z V_{2}+\cdots+z^{l-1} V_{l}\right)
$$


where $V_{1}, \ldots, V_{l}$ are $k l \times k$ matrices defined by

$$
\left[\begin{array}{llll}
V_{1} & V_{2} & \cdots & V_{l}
\end{array}\right]=\left(\operatorname{col}\left[X T_{1}^{j}\right]_{j=0}^{l-1}\right)^{-1},
$$

and

$$
\mathcal{P}(z)=z^{l}-\left(W_{1}+z W_{2}+\cdots+z^{l-1} W_{l}\right) T_{2}^{l} Y
$$

where $W_{1}, \ldots, W_{l}$ are $k \times k l$ matrices defined by

$$
\operatorname{col}\left[W_{j}\right]_{j=1}^{l}=\left[\begin{array}{llll}
Y & T_{2} Y & \cdots & T_{2}^{l-1} Y
\end{array}\right]^{-1} .
$$

These representations are called right and left canonical forms of $\mathcal{P}(z)$, respectively.

An important special case is the spectral triple $\left(Q, C_{\mathcal{P}}, R\right)$ given by

$$
Q^{T}=\left[\begin{array}{c}
0 \\
\vdots \\
0 \\
I_{k}
\end{array}\right], \quad C_{\mathcal{P}}=\left[\begin{array}{ccccc}
-A_{l-1} & -A_{l-2} & \cdots & -A_{1} & -A_{0} \\
I_{k} & 0 & \cdots & \cdots & 0 \\
0 & I_{k} & \ddots & & \vdots \\
\vdots & \ddots & \ddots & \ddots & \vdots \\
0 & \cdots & 0 & I_{k} & 0
\end{array}\right], \quad R=\left[\begin{array}{c}
I_{k} \\
0 \\
\vdots \\
0
\end{array}\right],
$$

where $C_{\mathcal{P}}$ is called the companion matrix of $\mathcal{P}(z),\left(Q, C_{\mathcal{P}}\right)$ is a right spectral pair and $\left(C_{\mathcal{P}}, R\right)$ is a left spectral pair of $\mathcal{P}(z)$. Then there exist $(k l \times k l)$ matrix polynomials $E(z)$ and $F(z)$ with constant nonzero determinant such that $E(z)(z-$ $\left.C_{\mathcal{P}}\right) F(z)=\mathcal{P}(z) \oplus I_{k(l-1)}$ for every $z \in \mathcal{C}$, cf. [11]. As a result, $\mathcal{P}(z)$ and $C_{\mathcal{P}}$ have the same eigenvalues and the same Jordan structure at each eigenvalue.

\section{Block-tridiagonal Toeplitz matrices}

Let $A=\left(A_{i-j}\right)_{i, j \in \mathbb{Z}}$ be a bi-infinite block tridiagonal Toeplitz matrix, where $A_{1}, A_{0}$ and $A_{-1}$ are $k \times k$ matrices and $A_{s}=0$ for $s \neq-1,0,1$.

We consider the factorization

(5.1) $A=L D U$

where $D=\left(D_{i-j}\right)_{i, j \in \mathbb{Z}}$ is a block diagonal Toeplitz matrix with $k \times k$ entries $D_{0}=\mathcal{D}_{k}$, and $L=\left(L_{i-j}\right)_{i, j \in \mathbb{Z}}$ and $U=\left(U_{i-j}\right)_{i, j \in \mathbb{Z}}$ are block bi-diagonal Toeplitz matrices with $k \times k$ entries $L_{1}=-\mathcal{L}_{k}^{T}, U_{-1}=-\mathcal{U}_{k}, L_{0}=U_{0}=I_{k}$ (the $k \times k$ identity matrix) and $L_{s}=U_{-s}=0$ for $s \neq 0,1$. The matrices $L, U$ and $D$ are factors in the factorization (5.1) if and only if $\mathcal{L}_{k}, \mathcal{U}_{k}$ and $\mathcal{D}_{k}$ satisfy the relations

$$
\left\{\begin{array}{l}
A_{0}=\mathcal{L}_{k}^{T} \mathcal{D}_{k} \mathcal{U}_{k}+\mathcal{D}_{k} \\
A_{l}=-\mathcal{L}_{k}^{T} \mathcal{D}_{k} \\
A_{-1}=-\mathcal{D}_{k} \mathcal{U}_{k} .
\end{array}\right.
$$

This factorization, which exists under very general hypotheses, is not unique. We are especially interested in giving an efficient method for finding the unique 
factorization such that $L$ and $U$ are boundedly invertible on the Hilbert space $l_{2}(\mathbb{Z})$ and, further, $L^{-1}$ and $\left(U^{T}\right)^{-1}$ are lower triangular block Toeplitz matrices.

In the following, we shall give the necessary and sufficient conditions for the existence of such a factorization. The proof is constructive, as it contains a numerical method for obtaining the factorization that we are looking for.

We shall assume that either $A_{1}$ or $A_{-1}$ is nonsingular. First suppose that $A_{1}$ is nonsingular. Now let the function

$$
\Sigma(z)=z A_{1}+A_{0}+z^{-1} A_{-1}
$$

be the symbol associated to the matrix $A$. Then $\mathcal{P}(z):=z A_{1}^{-1} \Sigma(z)$ is a monic $k \times k$ matrix polynomial of degree 2 . Now let $\Gamma$, with $0 \notin \Gamma$, be a simple closed oriented rectifiable Jordan curve dividing the complex plane into an interior domain $\Omega_{+}$ with $0 \in \Omega_{+}$and an exterior domain $\Omega_{-}$with $\infty \in \Omega_{-}$, and assume that $z \Sigma(z)$ (and hence $\mathcal{P}(z)$ ) does not have eigenvalues on $\Gamma$. Then $(5.1)$ is valid if and only if

$$
\Sigma(z)=\left(I_{k}-z \mathcal{L}_{k}\right)^{T} \mathcal{D}_{k}\left(I_{k}-z^{-1} \mathcal{U}_{k}\right)
$$

Moreover, as $A_{1}$ is nonsingular, it follows from $A_{1}=-\mathcal{L}_{k}^{T} \mathcal{D}_{k}$ that both $\mathcal{L}_{k}$ and $\mathcal{D}_{k}$ are nonsingular.

Let $\lambda_{1}, \ldots, \lambda_{s}$ be the distinct eigenvalues of $z \Sigma(z)$ in $\Omega_{-}$, let $\mu_{1}, \ldots, \mu_{t}$ be the distinct eigenvalues of $z \Sigma(z)$ in $\Omega_{+}$, put $z_{j}=\lambda_{j}$ for $j=1, \ldots, s$ and $z_{j}=\mu_{j-s}$ for $j=s+1, \ldots, s+t$, and let $m_{j}, j=1, \ldots, s+t$ denote the multiplicity of $z_{j}$ as a zero of $\operatorname{det}(z \Sigma(z))$. Then $m_{1}+\cdots+m_{s+t}=2 k$. The main result of this paper is the following theorem, which gives a constructive proof of the existence of the factorization (5.2).

THEOREM 5.1. A factorization (5.2) of $\Sigma(z)$, where $\mathcal{U}_{k}$ has its eigenvalues in $\Omega_{+}$ and $\mathcal{L}_{k}^{-1}$ has its eigenvalues in $\Omega_{-}$, exists if and only if

$$
m_{1}+\cdots+m_{s}=k=m_{s+1}+\cdots+m_{s+t}
$$

Whenever this factorization exists, it is unique and it is called the spectral factorization of $A$.

PROOF. As $\mathcal{D}_{k}$ is nonsingular, if $\Sigma(z)$ has the factorization (5.2), then the eigenvalues of $z \Sigma(z)$ are given by the union of the sets of eigenvalues of $\mathcal{U}_{k}$ and $\mathcal{L}_{k}^{-1}$. Hence, if the eigenvalues of $\mathcal{U}_{k}$ and $\mathcal{L}_{k}^{-1}$ are in $\Omega_{+}$and $\Omega_{-}$, respectively, relation (5.3) is satisfied.

Now suppose that relation (5.3) is true. We shall prove that the factorization (5.2) holds and that $\mu_{1}, \mu_{2}, \ldots, \mu_{t}$ are the eigenvalues of $\mathcal{U}_{k}$ and $\lambda_{1} \lambda_{2}, \ldots, \lambda_{s}$ are the eigenvalues of $\mathcal{L}_{k}^{-1}$. As in (4.3), we now construct the right spectral pair $\left(X_{r}, T_{r}\right)$ of $\mathcal{P}(z)$ by using its Jordan chains (which are also the Jordan chains of $z \Sigma(z))$ and the right spectral pair $\left(X_{l}, T_{l}\right)$ of $\left(A_{1}^{T}\right)^{-1} \mathcal{P}(z)^{T} A_{1}^{T}$ using its Jordan chains (which are also the Jordan chains of $z \Sigma(z)^{T}$ ), where the distinct eigenvalues 
$z_{1}, \ldots, z_{s+t}$ of $z \Sigma(z)$ have been ordered by decreasing modulus. Then, denoting the $m \times m$ upper triangular Jordan block with diagonal entries $\lambda$ by $J_{m}(\lambda)$ and partitioning the $k \times 2 k$ matrices $X_{l}$ and $X_{\tau}$ into $k \times k$ blocks, we have

$$
\begin{aligned}
& X_{l}=\left[\begin{array}{ll}
V & ?
\end{array}\right], \quad X_{\tau}=\left[\begin{array}{ll}
? & W
\end{array}\right], \\
& T_{l}=T_{r}=\operatorname{diag}\left(J_{m_{1}}\left(\lambda_{1}\right), \ldots, J_{m_{s}}\left(\lambda_{s}\right), J_{m_{s+1}}\left(\mu_{1}\right), \ldots, J_{m_{s+t}}\left(\mu_{t}\right)\right),
\end{aligned}
$$

where $V$ and $W$ are nonsingular $k \times k$ matrices and the $k \times k$ matrices at the question marks are irrelevant. Writing

$$
\begin{aligned}
& \Lambda_{L}^{-1}=\operatorname{diag}\left(J_{m_{1}}\left(\lambda_{1}\right), \ldots, J_{m_{s}}\left(\lambda_{s}\right)\right), \\
& \Lambda_{M}=\operatorname{diag}\left(J_{m_{s+1}}\left(\mu_{1}\right), \ldots, J_{m_{s+\ell}}\left(\mu_{t}\right)\right),
\end{aligned}
$$

we obtain the factorization (5.2) by defining

$$
\mathcal{L}_{k}=V \Lambda_{L} V^{-1}, \quad \mathcal{U}_{k}=W \Lambda_{M} W^{-1}, \quad \mathcal{D}_{k}=-\left(\mathcal{L}_{k}^{T}\right)^{-1} A_{1}
$$

Indeed, put

$$
\hat{A}_{0}=\mathcal{L}_{k}^{T} \mathcal{D}_{k} \mathcal{U}_{k}+\mathcal{D}_{k}=-A_{1} \mathcal{U}_{k}+\mathcal{D}_{k}, \quad \hat{A}_{-1}=-\mathcal{D}_{k} \mathcal{U}_{k}
$$

Then for $z \in \mathbb{C} \backslash\{0\}$ we have

$$
\hat{\Sigma}(z):=\left(I_{k}-z \mathcal{L}_{k}\right)^{T} \mathcal{D}_{k}\left(I_{k}-z^{-1} \mathcal{U}_{k}\right)=z A_{1}+\hat{A}_{0}+z^{-1} \hat{A}_{-1} .
$$

Now, letting $w_{0}, w_{1}, \ldots, w_{m-1}$ be a Jordan chain of $z \Sigma(z)$ corresponding to the eigenvalue $\mu_{j}$, by virtue of (4.1) we have

$$
\mu_{j} \Sigma\left(\mu_{j}\right) w_{p}+\left(2 \mu_{j} A_{1}+A_{0}\right) w_{p-1}+A_{1} w_{p-2}=0
$$

where $p=0,1, \ldots, m-1$ and $w_{-2}=w_{-1}=0$. Furthermore, in view of the definition of $\mathcal{U}_{k}$ given in (5.4), using (4.1) for $W(z)=z-\mathcal{U}_{k}$ we get

$$
\left(\mu_{j}-\mathcal{U}_{k}\right) w_{p}=-w_{p-1}
$$

where $p=0,1, \ldots, m-1$. The result of the computation

$$
\begin{aligned}
\mu_{j} \hat{\Sigma}\left(\mu_{j}\right) w_{p}+\left(2 \mu_{j} A_{1}+\hat{A}_{0}\right) w_{p-1}+A_{1} w_{p-2} \\
=\left(I_{k}-\mu_{j} \mathcal{L}_{k}\right)^{T} \mathcal{D}_{k}\left(\mu_{j}-\mathcal{U}_{k}\right) w_{p}+\mu_{j} A_{1} w_{p-1}+A_{1}\left(\mu_{j}-\mathcal{U}_{k}\right) w_{p-1} \\
\quad+\left(\hat{A}_{0}+A_{1} \mathcal{U}_{k}\right) w_{p-1}+A_{1} w_{p-2} \\
=-\left(I_{k}-\mu_{j} \mathcal{L}_{k}\right)^{T} \mathcal{D}_{k} w_{p-1}+\mu_{j} A_{1} w_{p-1}-A_{1} w_{p-2}+\mathcal{D}_{k} w_{p-1}+A_{1} w_{p-2} \\
=\mu_{j}\left(\mathcal{L}_{k}^{T} \mathcal{D}_{k}+A_{1}\right) w_{p-1}=0
\end{aligned}
$$

now implies that $w_{0}, \ldots, w_{m-1}$ is also a Jordan chain of $z \hat{\Sigma}(z)$ corresponding to the eigenvalue $\mu_{j}$. Next, letting $v_{0}, v_{1}, \ldots, v_{m-1}$ be a Jordan chain of $z \Sigma(z)^{T}$ corresponding to the (nonzero) eigenvalue $\lambda_{j}$, by virtue of (4.1) we have

$$
\lambda_{j} \Sigma\left(\lambda_{j}\right)^{T} v_{p}+\left(2 \lambda_{j} A_{1}^{T}+A_{0}^{T}\right) v_{p-1}+A_{1}^{T} v_{p-2}=0
$$


where $p=0,1, \ldots, m-1$ and $v_{-2}=v_{-1}=0$. Furthermore, in view of the definition of $\mathcal{L}_{k}$ given in (5.4), using (4.1) for $W(z)=z-\mathcal{L}_{k}^{-1}$ we obtain

$$
\left(\lambda_{j}-\mathcal{L}_{k}^{-1}\right) v_{p}=-v_{p-1}
$$

where $p=0,1, \ldots, m-1$. The result of the computation

$$
\begin{aligned}
& \lambda_{j} \hat{\Sigma}\left(\lambda_{j}\right)^{T} v_{p}+\left(2 \lambda_{j} A_{1}^{T}+\hat{A}_{0}^{T}\right) v_{p-1}+A_{1}^{T} v_{p-2} \\
&=-\left(\lambda_{j}-\mathcal{U}_{k}^{T}\right) \mathcal{D}_{k}^{T} \mathcal{L}_{k}\left(\lambda_{j}-\mathcal{L}_{k}^{-1}\right) v_{p}+\left(\lambda_{j}-\mathcal{U}_{k}^{T}\right) A_{1}^{T} v_{p-1} \\
& \quad+A_{1}^{T}\left(\lambda_{j}-\mathcal{L}_{k}^{-1}\right) v_{p-1}+A_{1}^{T} v_{p-2} \\
&=\left(\lambda_{j}-\mathcal{U}_{k}^{T}\right) \mathcal{D}_{k}^{T} \mathcal{L}_{k} v_{p-1}+\left(\lambda_{j}-\mathcal{U}_{k}^{T}\right) A_{1}^{T} v_{p-1}-A_{1}^{T} v_{p-2}+A_{1}^{T} v_{p-2} \\
&=\left(\lambda_{j}-\mathcal{U}_{k}^{T}\right)\left(\mathcal{D}_{k}^{T} \mathcal{L}_{k}+A_{1}^{T}\right) v_{p-1}=0
\end{aligned}
$$

now implies that $v_{0}, \ldots, v_{m-1}$ is also a Jordan chain of $z \hat{\Sigma}(z)^{T}$ corresponding to the eigenvalue $\lambda_{j}$. Consequently, subtracting (5.6) from (5.5) and (5.8) from (5.7), respectively, we obtain

$$
\begin{aligned}
& {\left[\mu_{j}\left(A_{0}-\hat{A}_{0}\right)+\left(A_{-1}-\hat{A}_{-1}\right)\right] w_{p}+\left(A_{0}-\hat{A}_{0}\right) w_{p-1}=0,} \\
& {\left[\lambda_{j}\left(A_{0}-\hat{A}_{0}\right)^{T}+\left(A_{-1}-\hat{A}_{-1}\right)^{T}\right] v_{p}+\left(A_{0}-\hat{A}_{0}\right)^{T} v_{p-1}=0}
\end{aligned}
$$

where $p=0,1, \ldots, m-1$. The first of these equations can be written as

$$
\begin{aligned}
& \left(A_{-1}-\hat{A}_{-1}\right)\left[\begin{array}{llll}
w_{0} & w_{1} & \cdots & w_{m-1}
\end{array}\right] \\
& =-\left(A_{0}-\hat{A}_{0}\right)\left[\begin{array}{llll}
w_{0} & w_{1} & \cdots & w_{m-1}
\end{array}\right] J_{m}\left(\mu_{j}\right)
\end{aligned}
$$

which implies $\left(A_{-1}-\hat{A}_{-1}\right) W=-\left(A_{0}-\hat{A}_{0}\right) W \Lambda_{M}$ and hence

$$
\left(A_{-1}-\hat{A}_{-1}\right)=-\left(A_{0}-\hat{A}_{0}\right) \mathcal{U}_{k} \text {. }
$$

In the same way we get $\left(A_{-1}-\hat{A}_{-1}\right)^{T} V=-\left(A_{0}-\hat{A}_{0}\right)^{T} V \Lambda_{L}^{-1}$ and hence $(5.10)\left(A_{-1}-\hat{A}_{-1}\right)^{T}=-\left(A_{0}-\hat{A}_{0}\right)^{T} \mathcal{L}_{k}^{-1}$.

From (5.9) and (5.10) one immediately gets

$$
\left(A_{0}-\hat{A}_{0}\right) \mathcal{U}_{k}=\left(\mathcal{L}_{k}^{T}\right)^{-1}\left(A_{0}-\hat{A}_{0}\right)=-\left(A_{-1}-\hat{A}_{-1}\right) .
$$

Since $\mathcal{U}_{k}$ and $\left(\mathcal{L}_{k}^{T}\right)^{-1}$ do not have eigenvalues in common, the latter identity implies $A_{0}-\hat{A}_{0}=A_{-1}-\hat{A}_{-1}=0$, or in other words $A_{0}=\hat{A}_{0}$ and $A_{-1}=\hat{A}_{-1}$. Hence the matrix polynomials $z \Sigma(z)$ and $z \hat{\Sigma}(z)$ coincide.

We now conclude that $\Sigma(z)$ has the factorization (5.2) and hence that the bi-infinite matrix $A$ has the factorization (5.1). To find the linear factors in (5.2) it is sufficient to find either $\mathcal{U}_{k}$ or $\mathcal{L}_{k}$. Indeed, if $\mathcal{U}_{k}$ is known, then $\mathcal{D}_{k}=A_{0}+A_{1} \mathcal{U}_{k}$ and $\mathcal{L}_{k}=-\left(A_{1} \mathcal{D}_{k}^{-1}\right)^{T}$; if $\mathcal{L}_{k}$ is known, then $\mathcal{D}_{k}=A_{0}+\mathcal{L}_{k}^{T} C$ and $\mathcal{U}_{k}=-\mathcal{D}_{k}^{-1} C$.

A similar factorization can be obtained by assuming that $A_{-1}$ rather than $A_{1}$ is nonsingular. In that case one replaces $z$ by $z^{-1}$ and changes the roles of $A_{1}$ and $A_{-1}$ to arrive at analogous factorization results. 
We note that, starting from the eigenvalues of $z \Sigma(z)$, we can generate several $k \times k$ matrices $\mathcal{L}_{k}$ and $\mathcal{U}_{k}$ such that (5.2) is satisfied. For example, if the eigenvalues are all distinct we can obtain the triple $\mathcal{L}_{k}, \mathcal{U}_{k}$ and $\mathcal{D}_{k}$ in $\left(\begin{array}{c}2 k \\ k\end{array}\right)$ different ways. Moreover, although Theorem 5.1 concerns the spectral factorization of $A$, it does not directly provide information on the existence and the properties of $L^{-1}$ and $U^{-1}$. To this end we establish the following

THEOREM 5.2. If $\mathcal{L}_{k}, \mathcal{D}_{k}$ and $\mathcal{U}_{k}$ are constructed as previously specified and $\Gamma$ is the unit circle, then the matrices $L$ and $U$ are boundedly invertible on $l_{2}$ and their inverses $L^{-1}$ and $\left(U^{-1}\right)^{T}$ are lower triangular block Toeplitz matrices of the type $\left(T_{i-j}\right)_{i, j \in \mathbb{Z}}$ where $T_{s}=0$ for $s<0, T_{0}=I_{k}$ and $\sum_{j=0}^{\infty} r^{j}\left\|T_{j}\right\|<+\infty$ for some $r>1$.

Proof. If (5.2) holds for $\Gamma$ the unit circle, then $\mathcal{L}_{k}^{T}$ and $\mathcal{U}_{k}$ both have all of their eigenvalues within the open unit disk and hence the series $\sum_{j=0}^{\infty} z^{j}\left(\mathcal{L}_{k}^{T}\right)^{j}$ and $\sum_{j=0}^{\infty} z^{-j}\left(\mathcal{U}_{k}\right)^{j}$ converge in the norm uniformly in $z \in\left\{w \in \mathbb{C}: r^{-1} \leq|w| \leq r\right\}$ for some $r>1$. Moreover, their sums are the inverses of the factors $\left(I_{k}-z \mathcal{L}_{k}\right)^{T}$ and $\left(I_{k}-z^{-1} \mathcal{U}_{k}\right)$, respectively. As a result, the bi-infinite Toeplitz matrices having these sums as their symbols are the inverses of $L$ and $U$, and these inverses are bounded on $l_{2}$. The final part, where $T_{s}$ is either of $\left(\mathcal{L}_{k}^{T}\right)^{s}$ or $\left(\mathcal{U}_{k}\right)^{s}$ for $s \geq 0$, is immediate, because the spectral radii of both $\mathcal{L}_{k}^{T}$ and $\mathcal{U}_{k}$ are less than $1 / r$ for some $r>1$.

If the bi-infinite matrix $A$ is real and positive definite and the contour $\Gamma$ is the unit circle, then $A_{0}, A_{1}$ and $A_{-1}$ are real matrices such that $A_{1}^{T}=A_{-2}$ and $A_{0}$ is positive definite. Moreover, there exists a unique spectral factorization with real coefficients in which $\mathcal{U}_{k}=\mathcal{L}_{k}$ is nonsingular and $\mathcal{D}_{k}$ is positive definite. Indeed, (5.1) and $A=A^{T}$ imply that $\left(L^{T} U^{-1}\right)^{T} D=D^{T}\left(L^{T} U^{-1}\right)$ is a block diagonal matrix with invertible blocks. Then $L^{T} U^{-1}$ is the identity matrix and $D=D^{T}$, so that $L=U^{T}$ and $D$ is positive definite. Hence $\mathcal{L}_{k}=\mathcal{U}_{k}$ and $\mathcal{D}_{k}$ is positive definite.

\section{Convergence of finite sections}

Consider the aforementioned bi-infinite block tridiagonal Toeplitz matrix $A$. We define the semi-infinite block Toeplitz matrices $A_{+}$and $A_{-}$as follows:

(6.1) $A_{+}=\left[\begin{array}{ccccc}A_{0} & A_{-1} & & & \\ A_{1} & A_{0} & A_{-1} & & \\ & A_{1} & A_{0} & A_{-1} & \\ & & \ddots & \ddots & \ddots\end{array}\right], A_{-}=\left[\begin{array}{ccccc}A_{0} & A_{1} & & & \\ A_{-1} & A_{0} & A_{1} & & \\ & A_{-1} & A_{0} & A_{1} & \\ & & \ddots & \ddots & \ddots\end{array}\right]$.

Then $A_{+}$(resp. $A_{-}$) are boundedly invertible on the Hilbert space $l_{2}$ of square summable complex sequences $\left(x_{i}\right)_{i=0}^{\infty}$ if and only if the symbol $\Sigma(z)=z A_{1}+A_{0}+$ $z^{-1} A_{-1}$ (resp., $\Sigma\left(z^{-1}\right)=z A_{-1}+A_{0}+z^{-1} A_{1}$ ) has the factorization (5.2) (resp. 
$\left.\Sigma\left(z^{-1}\right)=\left(I_{k}-z \tilde{\mathcal{L}}_{k}\right)^{T} \tilde{\mathcal{D}}_{k}\left(I_{k}-z^{-1} \tilde{\mathcal{U}}_{k}\right)\right)$ where all of the eigenvalues of $\mathcal{L}_{k}$ and $\mathcal{U}_{k}$ (resp. $\tilde{\mathcal{L}}_{k}$ and $\tilde{\mathcal{U}}_{k}$ ) have absolute value less than 1 [9, Theorem XXIV 4.1]. In other words, the block Toeplitz matrices $A_{+}$and $A_{-}$are boundedly invertible on $l_{2}$ if and only if there exist $k \times k$ matrices $\mathcal{L}_{k}, \tilde{\mathcal{L}}_{k}, \mathcal{U}_{k}$ and $\overline{\mathcal{U}}_{k}$ with all of their eigenvalues having modulus less than 1 and nonsingular $k \times k$ matrices $\mathcal{D}_{k}$ and $\tilde{\mathcal{D}}_{k}$ such that

$$
\Sigma(z)=\left(I_{k}-z \mathcal{L}_{k}\right)^{T} \mathcal{D}_{k}\left(I_{k}-z^{-1} \mathcal{U}_{k}\right)=\left(I_{k}-z^{-1} \tilde{\mathcal{L}}_{k}\right)^{T} \tilde{\mathcal{D}}_{k}\left(I_{k}-z \tilde{\mathcal{L}}_{k}\right)
$$

The factors in (6.2) can be found by applying the algorithm of the preceding subsection to $\Sigma(z)$ and to $\Sigma\left(z^{-1}\right)$.

Now we define on $l_{2}$ the projections $P_{n}$ of rank $n k$ as follows: $P_{n}\left(x_{i}\right)_{i=0}^{\infty}=$ $\left(x_{0}, x_{1}, \ldots, x_{n k}, 0,0,0, \ldots\right)$. Define the $(n k) \times(n k)$ matrices $A_{n+}$ and $A_{n-}$ as the corresponding left upper blocks of $A_{+}$and $A_{-}$, respectively. Then, according to Theorem 2.1 of [10] (also $[14,24]$ ), the matrices $A_{n+}$ and $A_{n-}$ are nonsingular for sufficiently large $n$ and for every $\mathrm{y} \in l_{2}$ the vector $\left(A_{n+}^{-1} \mathrm{y}, 0,0,0, \ldots\right)$ converges to $A_{+}^{-1} \mathbf{y}$ in the norm of $l_{2}$. Similarly, for every $\mathrm{y} \in l_{2}$ the vector $\left(A_{n-1}^{-1} \mathbf{y}, 0,0,0, \ldots\right)$ converges to $A_{-}^{-1} \mathrm{y}$ in the norm of $l_{2}$. In fact, these two convergence properties together are equivalent to the bounded invertibility of both $A_{+}$and $A_{-}$on $l_{2}$.

The semi-infinite matrices given by (6.1) have factorizations $A_{ \pm}=L_{ \pm} D_{ \pm} U_{ \pm}$ of the form (5.1) if and only if they are boundedly invertible on $l_{2}$. Then, for $n$ large enough, the matrices $A_{n \pm}$ corresponding to their $(n k) \times(n k)$ left upper corners have factorizations of the form

$$
A_{n+}=L_{n+} D_{n+} U_{n+}, \quad A_{n-}=L_{n-} D_{n-} U_{n-},
$$

where $D_{n+}$ and $D_{n-}$ are block diagonal matrices and $L_{n+}, L_{n-}, U_{n+}^{T}$ and $U_{n-}^{T}$ are lower block triangular matrices having $I_{k}$ as their diagonal blocks. Moreover, writing $\mathcal{J}_{n}[T]$ as the semi-infinite matrix obtained from the $(n k) \times(n k)$ matrix $T$ by adding zero entries, the extended $(n k) \times(n k)$ matrices $\mathcal{J}_{n}\left[L_{n \pm}\right], \mathcal{J}_{n}\left[L_{n \pm}^{-1}\right]$, $\mathcal{J}_{n}\left[U_{n \pm}\right], \mathcal{J}_{n}\left[U_{n \pm}^{-1}\right], \mathcal{J}_{n}\left[D_{n \pm}\right]$ and $\mathcal{J}_{n}\left[D_{n \pm}^{-1}\right]$ converge to the respective semi-infinite matrices $L_{ \pm}, L_{ \pm}^{-1}, U_{ \pm}, U_{ \pm}^{-1}, D_{ \pm}$and $D_{ \pm}^{-1}$ in the strong operator topology as $n \rightarrow \infty$. To prove these convergence properties, one applies the theory of multiplicative $L U$-decompositions [9] with respect to the chain of orthogonal projections $\left\{0, P_{0}, P_{1}, \cdots, I_{l_{2}}\right\}$ to the semi-infinite matrices $A_{ \pm}$, where $I_{l_{2}}$ is the identity operator on $l_{2}$. Using the same multiplicative $L U$-decompositions with respect to the same chain of projections in the Hilbert space of complex sequences $\left(x_{i}\right)_{i=0}^{\infty}$ endowed with the weighted norm $\left(\sum_{i \in \mathbb{Z}_{+}} r^{2 i}\left|x_{i}\right|^{2}\right)^{1 / 2}$ for a suitable $r>1$, one proves that the above convergence properties also hold with respect to the weighted norm. Details will be given in a future paper.

Similar results hold for the $L D U$-factorization of a bi-infinite block tridiagonal Toeplitz matrix $A$. Indeed, let $l_{2}(\mathbb{Z})$ stand for the Hilbert space of square summable sequences indexed by the integers and let us define on $l_{2}(\mathbb{Z})$ the projection $P_{n}^{\leftrightarrow}$ of rank $(2 n+1) k$ as follows: $P_{n}^{\leftrightarrow}\left(x_{i}\right)_{i=-\infty}^{\infty}=\left(\cdots, 0,0, x_{-n}, \cdots, x_{n}, 0,0, \cdots\right)$. 
Next, define the $(2 n+1) k \times(2 n+1) k$ matrices $A_{n}$ as the corresponding central blocks of $A$. Then, according to [10], Theorem 3.1, applied for $R=S, A_{n}$ is nonsingular for sufficiently large $n$ and for every $\mathrm{y} \in l_{2}(\mathbb{Z})$ the vector $\left(\cdots, 0,0, A_{n}^{-1} \mathrm{y}\right.$, $0,0, \cdots)$ converges to $A^{-1} y$ in the norm of $l_{2}(\mathbb{Z})$. Actually, this convergence property is equivalent to the invertibility of both $A_{+}$and $A_{-}$on $l_{2}$. Moreover, for sufficiently large $n$ the matrices $A_{n}$ have a factorization of the form

$$
A=L_{n} D_{n} U_{n}
$$

where $D_{n}$ is a block diagonal matrix and $L_{n}$ and $U_{n}^{T}$ are lower block triangular matrices having $I_{k}$ as their diagonal blocks. Moreover, writing $\mathcal{J}_{n}^{\leftrightarrow}[T]$ as the biinfinite matrix obtained from the $(2 n+1) k \times(2 n+1) k$ matrix $T$ by adding zero entries, the extended matrices $\mathcal{J}_{n}^{\leftrightarrow}\left[L_{n}\right], \mathcal{J}_{n}^{\leftrightarrow}\left[L_{n}^{-1}\right], \mathcal{J}_{n}^{\leftrightarrow}\left[U_{n}\right], \mathcal{J}_{n}^{\leftrightarrow}\left[U_{n}^{-1}\right], \mathcal{J}_{n}^{\leftrightarrow}\left[D_{n}\right]$ and $\mathcal{J}_{n}^{\leftrightarrow}\left[D_{n}^{-1}\right]$ converge to the respective bi-infinite matrices $L, L^{-1}, U, U^{-1}, D$ and $D^{-1}$ in the strong operator topology as $n \rightarrow \infty$. The proof of these convergence properties follows from the theory of multiplicative $L U$-decompositions [9] with respect to a suitable chain of orthogonal projections.

When $A$ is real positive definite and hence $A_{0}$ is real positive definite and $A_{j}^{T}=A_{-j}$ for $j \geq 1, A_{+}$and $A_{-}$are both boundedly invertible on $l_{2}$, since the factorizations (6.2) both exist. In this case the unique solution of the equation $A \mathrm{x}=\mathrm{y}$ can be approximated as in the previous two paragraphs.

If $A$ is a positive definite scalar Toeplitz matrix (i.e., $k=1$ ) several interesting properties have been proved for the elements of the Cholesky factors of $A_{+}, A$, $A_{+}^{-1}$ and $A^{-1}[17]$. In particular, the inequality (3.3) shows that as $n \rightarrow \infty$ the elements of $\mathcal{J}_{n}\left[L_{n}\right]$ decay exponentially to the coefficients of the spectral factor $\gamma(z)$. Likewise, it has been proved in $[18,(2.16)]$ that as $n \rightarrow \infty$ the coefficients of $L_{+}^{-1}$ converge exponentially to those of $\gamma(z)^{-1}$.

Our numerical experiments suggest that these properties can be extended to the block case. We plan to explore this possibility in the near future.

\section{An application}

Let $P_{3}$ be a polynomial of degree 3 . A Hermite B-spline of order 2 and support $[0,2]$ is a function $B \in C^{1}[0,2]$ which satisfies the condition [21]

$$
B(x)= \begin{cases}P_{3}(x), & 0 \leq x \leq 1 \\ \pm P_{3}(2-x), & 1 \leq x \leq 2\end{cases}
$$

Now let $B_{1}$ and $B_{2}$ be the following second order Hermite B-splines:

$$
\begin{aligned}
& B_{1}(x)= \begin{cases}x^{2}(3-2 x), & 0 \leq x \leq 1 \\
(2 x-1)(x-2)^{2}, & 1<x \leq 2,\end{cases} \\
& B_{2}(x)= \begin{cases}x^{2}(x-1), & 0 \leq x \leq 1 \\
(x-1)(x-2)^{2}, & 1<x \leq 2 .\end{cases}
\end{aligned}
$$


As $B_{1}^{(\alpha)}(0)=B_{1}^{(\alpha)}(2)=0$ for $\alpha=0,1, B_{1}(1)=1$ and $B_{1}^{\prime}(1)=0, B_{2}^{(\alpha)}(0)=$ $B_{2}^{(\alpha)}(2)=0$ for $\alpha=0,1, B_{2}(1)=0$ and $B_{2}^{\prime}(1)=1$, we call them cardinal.

Furthermore, let $\mathrm{B}$ be the two-dimensional vector

$$
\mathrm{B}(x)=\left[\begin{array}{l}
B_{1}(x) \\
B_{2}(x)
\end{array}\right]
$$

and let $\mathbf{B}_{j}(x):=\mathbf{B}(x-j), j \in \mathbb{Z}$, be the $j$-th integer translate of $\mathbf{B}$. Now, among the functional vectors $\left\{\mathbf{B}_{j}\right\}$, we introduce the inner product

$$
\left\langle\mathbf{B}_{h}, \mathrm{~B}_{k}\right\rangle:=\left[\begin{array}{ll}
\left\langle B_{1}(\cdot-h), B_{1}(\cdot-k)\right\rangle & \left\langle B_{1}(\cdot-h), B_{2}(\cdot-k)\right\rangle \\
\left\langle B_{2}(\cdot-h), B_{1}(\cdot-k)\right\rangle & \left\langle B_{2}(\cdot-h), B_{2}(\cdot-k)\right\rangle
\end{array}\right]
$$

where the symbol $\langle\cdot, \cdot\rangle$ denotes the usual inner product in $L^{2}(\mathbb{R})$. According to this definition, $\mathbf{B}_{h}$ is orthonormal to $\mathbf{B}_{k}$ if and only if $\left\langle\mathbf{B}_{h}, \mathbf{B}_{k}\right\rangle=I_{2} \delta_{h k}$, where $I_{2}$ is the $2 \times 2$ identity matrix and $\delta_{h k}$ is the Kronecker symbol.

The vector B, as well as other kinds of vectors of Hermite B-splines, has been used in the construction of orthonormal multiwavelets, that is in the construction of vectors of wavelets [6].

Because the support of each component of the vector $\mathbf{B}_{j}$ is confined to the interval $[0,2]$, the bi-infinite matrix $G:=\left(\left\langle\mathrm{B}_{h}, \mathrm{~B}_{k}\right\rangle\right)_{h, k \in \mathbb{Z}}$ is a symmetric block tridiagonal Toeplitz matrix characterized by the $2 \times 2$ matrices

$$
G_{1}=\frac{1}{420}\left[\begin{array}{cc}
54 & 13 \\
-13 & -3
\end{array}\right], \quad G_{0}=\frac{1}{420}\left[\begin{array}{cc}
312 & 0 \\
0 & 8
\end{array}\right], \quad G_{-1}=\frac{1}{420}\left[\begin{array}{cc}
54 & -13 \\
13 & -3
\end{array}\right] \text {. }
$$

The eigenvalues of the corresponding symbol

$$
\Sigma(z)=z G_{1}+G_{0}+z^{-1} G_{-1}
$$

are the zeros of the equation

$$
z^{4}-72 z^{3}+262 z^{2}-72 z+1=0 .
$$

It is immediate that the symbol (7.2) is a $2 \times 2$ matrix polynomial which is Hermitian and positive definite on the unit circle and has two eigenvalues inside and two outside the unit circle. Hence, the method of Section 3 allows us to obtain the factorization of the symbol with respect to the unit circle and, consequently, the factorization of $G$. As $G$ is positive definite, so is $D$ and hence we can write $G=\hat{L} \hat{L}^{T}$, where $\hat{L}=L D^{1 / 2}$ is the Cholesky factor of $G$.

Suppose that $n$ is an integer such that $n \geq 2$ and $m:=n-2$. We consider the orthonormalization on $[0, n]$ of the sequence $\bar{B}_{0}, \mathbf{B}_{1}, \ldots, \mathbf{B}_{m}$ by a Gram-Schmidt process. In such a process, for the sake of simplicity, we still define the inner product among $\left\{\mathrm{B}_{i}\right\}$ by $(7.1)$, but the symbol $\langle\cdot, \cdot\langle$ represents now the usual inner product in $L^{2}[0, n]$. The process generates the vectors $\mathrm{O}_{0}^{n}, \mathrm{O}_{1}^{n}, \ldots, \mathrm{O}_{m}^{n}$ such that

$$
\left\langle\mathrm{O}_{r}^{n}, \mathrm{O}_{s}^{n}\right\rangle=I_{2} \delta_{r s}, \quad r, s=0,1, \ldots, m .
$$


This procedure is well-defined since the corresponding $(m+1) \times(m+1)$ block tridiagonal Gram matrix $\hat{G}_{(m+1)}$ is nonsingular. It is then straightforward to prove that the functional vectors $\left\{\mathrm{O}_{i}^{n}\right\}$ can be expressed as follows:

$$
\mathrm{O}_{i}^{n}(x)=\sum_{j=0}^{i}\left(\hat{L}_{m+1}^{-1}\right)_{i j} \mathrm{~B}_{j}(x), \quad i=0,1, \ldots, m,
$$

where $\hat{L}_{(m+1)}$ is the Cholesky factor of $\hat{G}_{(m+1)}$.

Now let

$$
\mathrm{O}(x)=\sum_{j=0}^{\infty} \hat{L}_{j}^{-} \mathrm{B}_{-j}(x)
$$

where $\left\{\hat{L}_{j}^{-}\right\}$is the sequence of $2 \times 2$ matrices defined by the series

$$
\mathcal{D}^{-1 / 2}\left(I_{k}-z \mathcal{L}_{k}\right)^{-1}=\sum_{j=0}^{\infty} \hat{L}_{j}^{-} z^{j}
$$

Furthermore, as a result of Theorem 5.2 , the norms $\left\|\hat{L}_{j}^{-}\right\|$decay exponentially as $j \rightarrow \infty$, i.e. there exist constants $c>0$ and $r \in(0,1)$ such that $\left\|\hat{L}_{j}^{-}\right\| \leq c r^{j}$ for all $j \in \mathbb{Z}_{+}$.

Our numerical experiments suggest that $\mathrm{O}(x)$ is the limiting profile of the sequence $\left\{\mathrm{O}_{i}^{n}\right\}$. Hence we conjecture that there exist constants $c>0$ and $\alpha \in$ $(0,1)$ such that for any $n \geq 2, m$ as above and $0 \leq i \leq m$

$$
\left\|\mathrm{O}_{i}^{n}(x)-\mathrm{O}_{i}(x)\right\| \leq c \alpha^{i}, \quad x \in \mathbb{R}_{+},
$$

where $\mathrm{O}_{i}(x):=\mathbf{O}(x-i)$ and $\|\mathbf{f}(x)\|=\max \left\{\left\|f_{1}(x)\right\|_{\infty},\left\|f_{2}(x)\right\|_{\infty}\right\}$. Figure 2 gives an idea of the behavior of the two components of $\mathrm{O}_{i}^{20}(x)$ for some values of $i$ and Figure 3 represents the graph of the components of $\mathrm{O}(x)$.
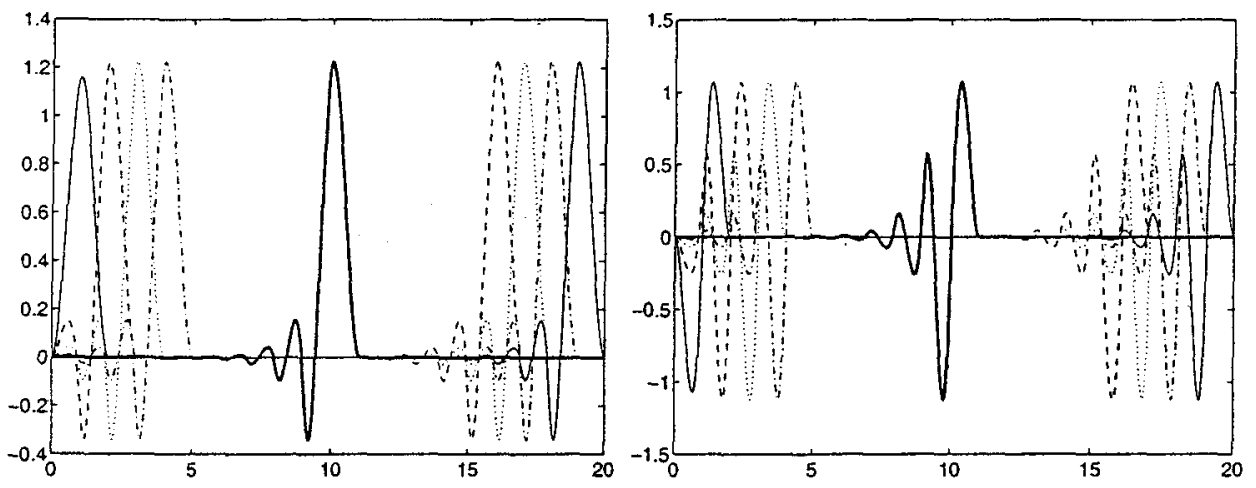

Figure 2: Components of $\mathrm{O}_{i}^{20}, i=0,1,2,3,9,15,16,17,18$ 


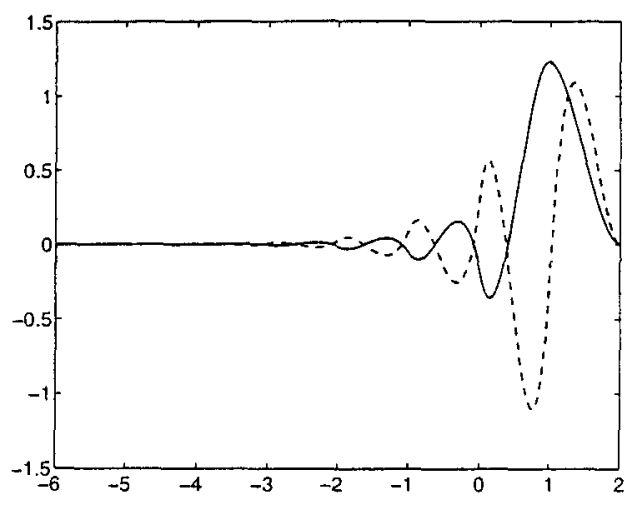

Figure 3: 2nd order Hermite O-splines

This is a special case of the more general problem concerning the asymptotic behavior of the Gram-Schmidt process for the orthonormalization of a large number of vectors of locally supported functions. We plan to study this problem in a next paper.

\section{Multi-index Toeplitz matrices}

As we have seen above, the orthonormalization of $B$-splines can be reduced to the $L D U$-factorization of a bi-infinite Toeplitz matrix. The generalization of this problem to vector-valued $\mathrm{B}$-splines leads to the $L D U$-factorization of bi-infinite block Toeplitz matrices. A different generalization occurs at the orthonormalization of $m$-dimensional B-splines, with $m \geq 2$. This problem can in principle be reduced to the $L D U$-factorization for Toeplitz matrices with $m$ indices where $m \geq 2$. In this section we study the definition, existence and stability under finite section truncation of the $L D U$-factorization of $m$-index bi-infinite Toeplitz matrices.

Let $l_{2}\left(\mathbb{Z}^{m}\right)$ be the Hilbert space of all square summable functions $x: \mathbb{Z}^{m} \rightarrow \mathbb{C}$ with norm

$$
\|x\|^{2}=\sum_{\alpha \in \mathbb{Z}^{m}}\left|x_{\alpha}\right|^{2}, \quad x=\left(x_{\alpha}\right)_{\alpha \in \mathbb{Z}^{m}}
$$

and inner product

$$
\langle x, y\rangle=x^{*} y=\sum_{\alpha \in \mathbb{Z}^{m}} \bar{x}_{\alpha} y_{\alpha}, \quad x=\left(x_{\alpha}\right)_{\alpha \in \mathbb{Z}^{m}}, \quad y=\left(y_{\alpha}\right)_{\alpha \in \mathbb{Z}^{m}}
$$

where $x^{*}=\left(\bar{x}_{\alpha}\right)_{\alpha \in \mathbb{Z}^{m}}$. Here we have written $x_{\alpha}$ instead of $x(\alpha)$.

Introducing the right shift $S_{k}$ by $S_{k}\left(x_{\alpha}\right)_{\alpha \in \mathbb{Z}^{m}}=\left(x_{\alpha-\gamma(k)}\right)_{\alpha \in \mathbb{Z}^{m}}, k=1, \ldots, m$, where $\gamma(k) \in \mathbb{Z}^{m}$ is the element of $\mathbb{Z}^{m}$ with $k$-th entry 1 and zero for the other entries, a bi-infinite Toeplitz matrix $A=\left(A_{\alpha-\beta}\right)_{\alpha, \beta \in \mathbb{Z}^{m}}$ is defined as any bounded 
linear operator $A$ on $l_{2}\left(\mathbb{Z}^{m}\right)$ that commutes with the right shifts $S_{1}, \cdots, S_{m}$. Such a Toeplitz matrix is said to be in the Wiener class if

$$
\sum_{\alpha \in \mathbb{Z}^{m}}\left|A_{\alpha}\right|<+\infty
$$

In that case we define the symbol by

$$
\hat{A}(z)=\sum_{\alpha \in \mathbb{Z}^{m}} A_{\alpha} z^{\alpha}, \quad z=\left(z_{1}, \cdots, z_{m}\right) \in \mathbb{T}^{m},
$$

where $z^{\alpha}=z_{1}^{\alpha_{1}} \cdots z_{m}^{\alpha_{m}}$ for $\alpha=\left(\alpha_{1}, \cdots, \alpha_{m}\right)$ and $\mathbb{T}^{m}=\left\{z=\left(z_{1}, \cdots, z_{m}\right) \in\right.$ $\left.\mathbb{C}^{m}:\left|z_{1}\right|=\cdots=\left|z_{m}\right|=1\right\}$ is the $m$-dimensional torus. Clearly, the symbol of a bi-infinite Toeplitz matrix in the Wiener class is continuous on $\mathbb{T}^{m}$. Using standard Banach algebra theory [ 9 , Chapter XXX] one sees immediately that the bi-infinite Toeplitz matrices in the Wiener class form a commutative $C^{*}$-algebra with respect to convolution [i.e., $(A * B)_{\alpha}=\sum_{\gamma \in \mathbb{Z}^{m}} A_{\alpha-\gamma} B_{\gamma}$ ] whose multiplicative linear functionals are exactly the maps $A \mapsto \hat{A}(z)$ for $z \in \mathbb{T}^{m}$. As a result, a biinfinite Toeplitz matrix $A$ in the Wiener class whose symbol $\hat{A}(z)$ does not vanish for $z \in \mathbb{T}^{m}$, has a bounded inverse in the Wiener class whose symbol is given by $1 / \hat{A}(z)$.

To define the $L D U$-factorization of a bi-infinite $m$-index Toeplitz matrix with $m \geq 2$ one needs a linear order $\preceq$ on $\mathbb{Z}^{m}$ which allows one to call a Toeplitz matrix $A$ lower (resp. upper) triangular if $A_{\alpha}=0$ for all $\alpha \in \mathbb{Z}^{m}$ with $\alpha \prec(0, \cdots, 0)$ (resp. $\alpha \succ(0, \cdots, 0)$ ). This linear order must have the following properties:

(a) $\alpha \preceq \beta \Longrightarrow \alpha+\gamma \preceq \beta+\gamma$, and

(b) $(\alpha \preceq \beta$ and $c \geq 0) \Longrightarrow c \alpha \preceq c \beta$.

The main problem is that for $m \geq 2$ such a so-called term ordering is by no means unique. In fact, the lexicographical order on $\mathbb{Z}^{m}$ with respect to any order of the "letters" $1, \cdots, m$ within the "alphabet" $\{1, \cdots, m\}$ will do. With respect to $\preceq$, we call $A=\left(A_{\alpha-\beta}\right)_{\alpha, \beta \in \mathbb{Z}^{m}}$ lower triangular if $A_{\alpha}=0$ for all $\alpha \in \mathbb{Z}^{m}$ with $\alpha \prec(0, \cdots, 0)$, upper triangular if $A_{\alpha}=0$ for all $\alpha \in \mathbb{Z}^{m}$ with $\alpha \succ(0, \cdots, 0)$, and diagonal if $A_{\alpha}=0$ for all $\alpha \in \mathbb{Z}^{m}$ different from $(0, \cdots, 0)$.

Consider the linear order $\preceq$ of $\mathbb{Z}^{m}$ as specified above. Let us study representations of $A$ in the form

(8.1) $A=L D U$,

where $L=\left(L_{\alpha-\beta}\right)_{\alpha, \beta \in \mathbb{Z}^{m}}$ is lower triangular with $L_{(0, \cdots, 0)}=1, U=\left(U_{\alpha-\beta}\right)_{\alpha, \beta \in \mathbb{Z}^{m}}$ is upper triangular with $U_{(0, \cdots, 0)}=1$, and $D=\left(D_{\alpha-\beta}\right)_{\alpha, \beta \in \mathbb{Z}^{m}}$ is a diagonal matrix. This factorization, which can be proven to exist under very general conditions on $A$, is not unique. To make it unique, we also require $D$ to be invertible (i.e., $D_{0}$ to be nonzero) and $L$ and $U$ to be boundedly invertible on $l_{2}\left(\mathbb{Z}^{m}\right)$ with inverses $L^{-1}$ and $U^{-1}$ that are lower and upper triangular matrices, respectively. In that case, 
$A$ has to be boundedly invertible on $l_{2}\left(\mathbb{Z}^{m}\right)$, but in general this is not sufficient for the existence of an $L D U$-factorization. A representation of $A$ in the form (8.1) where $L, D$ and $U$ have the above additional properties, is called an $L D U$ factorization of $A$. It is now easily seen that $L D U$-factorization of $A$ with factors and its inverses in the Wiener class amounts to the factorization

$$
\text { (8.2) } \hat{A}(z)=\hat{L}(z) D_{0} \hat{U}(z), \quad z \in \mathbb{T}^{m} \text {, }
$$

in terms of the corresponding symbols, where $D_{0}$ is a nonzero constant, $\hat{L}(z)-1$ and $\hat{L}(z)^{-1}-1$ have Fourier series where all terms proportional to $z^{\alpha}$ with $\alpha \prec$ $(0, \cdots, 0)$ vanish, and $\hat{U}(z)-1$ and $\hat{U}(z)^{-1}-1$ have Fourier series where all terms proportional to $z^{\alpha}$ with $\alpha \succ(0, \cdots, 0)$ vanish.

If $A$ is a bi-infinite Toeplitz matrix in the Wiener class and its symbol $\hat{A}(z)>$ 0 for all $z \in \mathbb{T}^{m}$, i.e., if $A$ is positive definite on $l_{2}\left(\mathbb{Z}^{m}\right)$, the factorization (8.2) exists. In that case, $D_{0}>0, \hat{U}(z)=\hat{L}\left(\bar{z}^{-1}\right)^{*}$, and therefore

$$
A(z)=\tilde{L}(z) \tilde{L}\left(\bar{z}^{-1}\right)^{*}, \quad \tilde{L}(z)=\hat{L}(z) \sqrt{D_{0}}
$$

leads to a Cholesky factorization of $A$.

Generalizing the existence theory for $L D U$-factorizations using the WienerHopf factorization theory for their symbols is not obvious if $m \geq 2$. First of all, there is no obvious geometrical criterion for the existence of the factorization (8.2) and the construction of the factors as in the case $m=1$ where the winding number turned out to be the key to the solution of the problem. Secondly, the factorization depends in an essential way on the choice of the linear order $\preceq$ on $\mathbb{Z}^{m}$.

The generalization of the previous results for $m=1$ will be based on the closely related theories of chains of projections in a Hilbert space $[15,9]$ and of nest algebras [8], which have the additional advantage of yielding an $L D U$ factorization theory for arbitrary bounded linear operators on $l_{2}\left(\mathbb{Z}^{m}\right)$ or on general separable Hilbert spaces. Before defining $L D U$-factorization with respect to a complete chain of orthogonal projections (using the language of [9, Chapter XXII]) or with respect to a nest (using the language of [8]), we first introduce the necessary preliminaries.

Let $H$ be a (real or complex) Hilbert space. By a nest $\mathcal{N}$ we mean a chain of closed subspaces containing $\{0\}$ and $H$ that is closed with respect to intersection and closed linear spans. For every $N \in \mathcal{N}, P_{N}$ denotes the orthogonal projection onto $N$. Then $\left\{P_{N}: N \in \mathcal{N}\right\}$ is a chain of orthogonal projections containing 0 and $I$ that is closed in the strong operator topology. By a jump we mean a pair $\left\{P_{N_{1}}, P_{N_{2}}\right\}$ where the nest does not contain subspaces between $N_{1}$ and $N_{2}$. The nest is maximal if and only if all jumps are one-dimensional. A chain of projections is called discrete if every nontrivial subspace $N \in \mathcal{N}$ has a jump on either side in the linear order, and continuous if there are no jumps at all.

Let us give some illustrative examples, characterized by one index $((1)-(3))$ and by $m$ indexes $((4)-(6))$. 
(1) Let $0=P_{0}, P_{1}, \cdots, P_{n}=I$ be a finite set of orthogonal projections on $H$ such that $\operatorname{Im} P_{j-1} \subset P_{j}(j=1, \cdots, n)$. This is called a finite chain.

(2) Let $H=l_{2}(\mathbb{N})$. Define $P_{0}=0, P_{+\infty}=I$, and

$$
P_{n}\left(x_{1}, \cdots, x_{n}, \cdots\right)=\left(x_{1}, \cdots, x_{n-2}, x_{n-1}, x_{n}, 0,0,0, \cdots\right)
$$

This sequence is a semi-infinite chain.

(3) Let $H=l_{2}(\mathbb{Z})$. Define $P_{-\infty}=0, P_{+\infty}=I$, and

$$
P_{n}\left(\cdots, x_{-1}, x_{0}, x_{1}, \cdots, x_{n}, \cdots\right)=\left(\cdots, x_{n-1}, x_{n}, 0, \cdots\right)
$$

This sequence is a bi-infinite chain.

(4) For $m \in \mathbb{N}$, consider $H=l_{2}\left(\mathbb{Z}^{m}\right)$, where $\mathbb{Z}^{m}$ is ordered lexicographically (by $\leq)$. For $j=1, \cdots, m$ and $\alpha=\left(\alpha_{1}, \cdots, \alpha_{j}\right) \in \mathbb{Z}^{j}$ we define $Q_{\alpha}$ by

$$
\begin{aligned}
& Q_{\alpha}\left(x_{\beta}\right)_{\beta \in \mathbb{Z}^{m}}=\left(y_{\alpha}\right)_{\beta \in \mathbb{Z}^{m}} \\
& y_{\beta}= \begin{cases}x_{\beta}, & \left(\beta_{1}, \cdots, \beta_{j}\right) \leq\left(\alpha_{1}, \cdots, \alpha_{j}\right) \\
0, & \left(\beta_{1}, \cdots, \beta_{j}\right)>\left(\alpha_{1}, \cdots, \alpha_{j}\right) .\end{cases}
\end{aligned}
$$

Then $0,\left\{Q_{\alpha}: \alpha \in \mathbb{Z}^{j}, j=1, \cdots, m\right\}$ and $I$ form a chain. We have

$$
Q_{\left(\alpha_{1}-1\right)} \leq Q_{\left(\alpha_{1}, \cdots, \alpha_{m}\right)} \leq \cdots \leq Q_{\left(\alpha_{1}, \alpha_{2}\right)} \leq Q_{\left(\alpha_{1}\right)}
$$

(5) In $\mathbb{Z}^{m}$ we define the term ordering

$$
\left(\alpha_{1}, \cdots, \alpha_{m}\right) \prec\left(\beta_{1}, \cdots, \beta_{m}\right) \Longleftrightarrow\left(\gamma_{1}, \cdots, \gamma_{m}\right)<\left(\delta_{1}, \cdots, \delta_{m}\right),
$$

where $\gamma_{j}=\alpha_{1}+\cdots+\alpha_{m}$ and $\delta_{j}=\beta_{1}+\cdots+\beta_{m}$.

For $j=1, \cdots, m$ and $\alpha=\left(\alpha_{1}, \cdots, \alpha_{j}\right) \in \mathbb{Z}^{j}$, define $P_{\alpha}$ by

$$
\begin{aligned}
& P_{\alpha}\left(x_{\beta}\right)_{\beta \in \mathbb{Z}^{m}}=\left(y_{\alpha}\right)_{\beta \in \mathbb{Z}^{m}} \\
& y_{\beta}= \begin{cases}x_{\beta}, & \left(\beta_{1}, \cdots, \beta_{j}\right) \preceq\left(\alpha_{1}, \cdots, \alpha_{j}\right) \\
0, & \left(\beta_{1}, \cdots, \beta_{j}\right) \succ\left(\alpha_{1}, \cdots, \alpha_{j}\right) .\end{cases}
\end{aligned}
$$

Then $0,\left\{P_{\alpha}: \alpha \in \mathbb{Z}^{j}, j=1, \cdots, m\right\}$ and $I$ form a chain. 
(6) Put $\mathbb{Z}_{+}^{m}=\left\{\alpha \in \mathbb{Z}^{m}: \alpha_{j} \geq 0, j=1, \cdots, m\right\}$. Consider $H=l_{2}\left(\mathbb{Z}_{+}^{m}\right)$ with the chain consisting of the projections $P_{\alpha}\left(\alpha_{j} \geq 0, j=1, \cdots, m\right)$ and $I$. This is a discrete chain.

Given the finite chain $\mathcal{P}=\left\{0=P_{0}, P_{1}, \cdots, P_{n}=I\right\}$ on the Hilbert space $H$, consider the bounded linear operator $A$ on $H$. Then $A$ is called diagonal if $P A=A P$ for every $P \in \mathcal{P}$; the diagonal part of $A$ is defined by $A_{\text {diagonal }}=$ $\sum_{j=1}^{n}\left(\Delta P_{j}\right) A\left(\Delta P_{j}\right)$, where $\Delta P_{j}=P_{j}-P_{j-1}(j=1, \cdots, n)$. Further, $A$ is called lower (resp. upper) triangular if $P A=P A P$ (resp. $A P=P A P$ ) for every $P \in \mathcal{P}$. Then by an $L D U$-factorization of $A$ with respect to the chain $\mathcal{P}$ we mean a representation of $A$ in the form (8.1), where $L, D$ and $U$ are boundedly invertible, $D$ is diagonal, $L$ and $L^{-1}$ are lower triangular with the identity operator as their diagonal parts, and $U$ and $U^{-1}$ are upper triangular with the identity operator as their diagonal parts. Moreover, if such a factorization exists, it is unique and the factors are given by

$$
\begin{aligned}
L & =\prod_{j=1}^{\hat{n}}\left[I-\Delta P_{j} K P_{j-1}\left(I-P_{j-1} K P_{j-1}\right)^{-1}\right] \\
U & =\prod_{j=1}^{n}\left[I-\left(I-P_{j-1} K P_{j-1}\right)^{-1} P_{j-1} K \Delta P_{j}\right] \\
D & =\sum_{j=1}^{n} \Delta P_{j}\left[I-K-K P_{j-1}\left(I-P_{j-1} K P_{j-1}\right)^{-1} K\right] \Delta P_{j} \\
L^{-1} & =I+\sum_{j=1}^{n} \Delta P_{j} K P_{j-1}\left(I-P_{j-1} K P_{j-1}\right)^{-1} \\
U^{-1} & =I+\sum_{j=1}^{n}\left(I-P_{j-1} K P_{j-1}\right)^{-1} P_{j-1} K \Delta P_{j} \\
D^{-1} & =\sum_{j=1}^{n} \Delta P_{j}\left(I-P_{j} K P_{j}\right)^{-1} \Delta P_{j},
\end{aligned}
$$

where $I$ is the identity operator, $K=I-A$, and the factors in the products determining $L$ (resp. $U$ ) are to be read from the right to the left (resp. from the left to the right). The existence of the bounded inverses $\left(I-P_{j-1} K P_{j-1}\right)^{-1}$ $(j=1, \cdots, n)$ is a necessary and sufficient condition for the existence of the $L D U$ factorization of $A$. In particular, if $A$ is positive definite on $H$ (i.e., if $\exists \kappa>0$ : $\langle A x, x\rangle \geq \kappa\|x\|^{2}$ for all $\left.x \in H\right)$, then $A$ has an $L D U$-factorization.

The definitions of diagonal, lower triangular and upper triangular operators and of $L D U$-factorizations, all with respect to a (closed) chain $\mathcal{P}$ of orthogonal projections, can be given by approximating the chain $\mathcal{P}$ by finite subchains and employing the above definitions. For instance, given the chain $\mathcal{P}=\{0=$ 
$\left.P_{0}, P_{1}, \cdots, I\right\}$ on $H=l_{2}(\mathbb{N})$ [cf. Example (1)] and the boundedly invertible operator $A=I-K$ on $H$, the $L D U$-factorization (8.1) of $A$ exists if and only if

$$
\sup _{j}\left\|\left(I-P_{j} K P_{j}\right)^{-1} P_{j}\right\|<+\infty
$$

and either of the series for $L^{-1}$ and $U^{-1}$ below converges strongly. Then the factors $L, D$ and $U$ and their inverses when they exist, are uniquely determined by $A$ and are given by infinite series and infinite products, which can be formally obtained by replacing $n$ by $\infty$ in (8.3). By a simple approximation argument one now easily proves $[8,9]$ that a positive definite operator $A$ on $l_{2}(\mathbb{N})$ admits an $L D U$-factorization and hence a Cholesky factorization $A=\mathrm{E} \mathrm{E}^{*}$ where $\mathrm{E}=L D^{1 / 2}$.

If $\mathcal{P}$ is a general (closed) chain of orthogonal projections on a Hilbert space $H$, one can define diagonal, lower triangular and upper triangular operators and $L D U$-factorizations by approximating the chain by finite subchains and utilizing the above definitions for finite chains. For the examples (1)-(6) of chains given above, one thus obtains expressions for the $L D U$-factors and their inverses that contain infinite series and infinite products that converge in the strong operator topology (cf. [9]). If the chain is uncountable, the infinite sums and infinite products are to be replaced by integrals and so-called multiplicative integrals, respectively.

The following result can be found in [8]. The proof for the special case of example (1) given in [9] is easily adapted to examples (2)-(6).

THEOREM 8.1. Let $A$ be positive definite on $H$, and let $\mathcal{P}$ be a countable chain of orthogonal projections in $H$ that is closed with respect to the strong operator topology. Then $A$ has a unique $L D U$-factorization as well as a Cholesky factorization with respect to $\mathcal{P}$.

Theorem 8.1 does not hold for uncountable chains. In fact, given un uncountable chain of orthogonal projections in $H$ that is closed with respect to the strong operator topology, then for every $\varepsilon>0$ there exists a compact operator $K$ with $\|K\|<\varepsilon$ such that $A=I-K$ is positive definite and does not have an $L D U$-factorization with respect to the chain.

Given the chain $\mathcal{P}=\left\{0=P_{0}, P_{1}, \cdots, I\right\}$ on $H=l_{2}(\mathbb{N})$ [cf. Example (1)], we have derived the expressions for the $L D U$-factors and their inverses of a semiinfinite matrix $A$ above, where the infinite series and infinite products converge strongly. When the summations and products run over $j=1, \cdots, n$ instead, one gets the $L D U$-factorization of the compression of $A$ to the range of $P_{n}$ with respect to the finite subchain $\mathcal{P}^{(n)}=\left\{0=P_{0}, P_{1}, \cdots, P_{n}, I\right\}$, as is apparent from the expressions for the $L D U$-factors and their inverses in the case of a finite chain. In other words, if $A$ has an $L D U$-factorization, its factors and their inverses can be obtained as the strong limits of the $L D U$-factors and their inverses for the $n \times n$ finite compression of $A$ as $n \rightarrow+\infty$. Similar results hold for $L D U$-factorizations 
with respect to arbitrary countable (closed) chains. It is not obvious how to obtain results on the rate of convergence.

Finally, $L D U$-factorization for bi-infinite and semi-infinite Toeplitz matrices are easily obtained from the general $L D U$-factorization results as follows. Consider $H=l_{2}(\mathbb{Z})$ and let $S$ be the right shift. Then a bi-infinite matrix $A$ is a Toeplitz matrix if and only if $A S=S A$. Since similarity using $S$ maps the sets of lower triangular, diagonal and upper triangular matrices onto themselves, the factors in the $L D U$-factorization of a Toeplitz matrix are themselves Toeplitz matrices. Analogously, consider $H=l_{2}\left(\mathbb{Z}^{m}\right)$ and let $S_{1}, \cdots, S_{m}$ be the elementary right shifts. Then a bi-infinite matrix $A$ is a Toeplitz matrix if and only if $A S_{j}=S_{j} A(j=1, \cdots, m)$. Since the similarities using $S_{1}, \cdots, S_{m}$ map the sets of lower triangular, diagonal and upper triangular matrices onto themselves, the factors in the $L D U$-factorization of a Toeplitz matrix are themselves Toeplitz matrices.

\section{REFERENCES}

[1] K. Amaratunga, A fast matlab routine for calculating Daubechies filters, Wavelet Digest 4(4), (1995).

[2] F.L. BAUER, Ein direktes Iterations Verfahren zur Hurwitz-zerlegung eines Polynoms, Arch. Elektr. Uebertragung 9, (1955) 285-290.

[3] F.L. BAUER, Beiträge zur Entwicklung numerischer Verfahren für programmgesteuerte Rechenanlagen, ii. Direkte Faktorisierung eines Polynoms, Sitz. Ber. Bayer. Akad. Wiss., (1956) 163-203.

[4] B.P. Bogert, M.J.R. Healy, J.W. Tukey, The quefrency alanysis of time serier for echoes: cepstrum pseudo-autocovariance, cross-cepstrum and saphe cracking, In M. Rosenblatt, editor, Proc. Symposium Time Series Analysis, pages 209-243, New York, 1963. John Wiley and Sons.

[5] A. Calderón, F. Spitzer, H. Widom, Inversion of Toeplitz matrices, Illinois J. Math. 3, (1959) 490-498.

[6] M. Cotronel, Multiwavelets: Analisi Teorica ed Algoritmi, $\mathrm{PhD}$ thesis, University of Messina, Italy, 1996.

[7] I. Daubechies, Ten Lectures on Wavelets, volume 61 of CBMS-NSF Regional Conference Series in Applied Mathematics, SIAM, Philadelphia, PA, 1992.

[8] K.R. Davidson, Nest Algebras, volume 191 of Research Notes in Mathematics, Pitman, London, 1988.

[9] I. Gohberg, S. Goldberg, M.A. KaAshoek, Classes of Linear Operators, Vol. II, volume 63 of Operator Theory: Advances and Applications, Birkhäuser, Basel-Boston, 1993. 
[10] I. Gohberg AND M.A. KAASHOEk, Projection methods for block Toeplitz operators with operator-valued symbols, In E.L. Basor and I. Gohberg, editors, Toeplitz Operators and Related Topics. The Harold Widom Anniversary Volume Workshop on Toeplitz and Wiener-Hopf Operators, Santa Cruz, California, September 20-22, 1992, volume 71 of Operator Theory: Advances and Applications, pages 79-104, Basel-Boston, 1994. Birkhäuser.

[11] I. Gohberg, P. LANCASTer, L. Rodman, Spectral analysis of matrix polynomials. I. Canonical forms and divisors, Linear Algebra and Applications 20, (1978) 1-44.

[12] I. Gohberg, P. Lancaster, L. Rodman, Matrix Polynomials, Academic Press, New York, 1982.

[13] I. Gohberg AND E.I. Sigal, An operator generalization of the logarithmic residue theorem and the theorem of Rouché, Mat. Sbornik 84(4), (1971) 607-629 (Russian, translated in Math. USSR Sbornik 13(4), (1971) 603-625).

[14] I.C. Gohberg, I.A. Feldman, Convolution Equations and Projection Methods for their Solution, volume 41 of Transl. Math. Monographs, AMS, Providence, R.I., 1974.

[15] I.C. Gohberg AND M.G. KREIN, Theory and Applications of Volterra Operators in Hilbert Space, volume 24 of Transl. Math. Monographs, AMS, Providence, R.I., 1970.

[16] G.H. Golub And C.F. Van Loan, Matrix Computations, The John Hopkins University Press, Baltimore, third edition, 1996.

[17] T.N.T. Goodman, C.A. Micchelli, G. Rodriguez, S. Seatzu, On the Cholesky factorization of the Gram matrix of locally supported functions, BIT 35(2), (1995) 233-257.

[18] T.N.T. Goodman, C.A. Micchelli, G. Rodriguez, S. Seatzu, On the limiting profile of exponentially decaying functions. Preprint, 1996.

[19] T.N.T. Goodman, C.A. Micchelli, G. Rodriguez, S. Seatzu, Spectral factorization of Laurent polynomials. Adv. in Comp. Math. (1997). To appear.

[20] M.G. KREIN, Integral equations on the half-line with kernel depending upon the difference of the arguments. Uspehi Mat. Nauk. 13(5), (1958) 3-120 (Russian, translated in AMS Translations 22, (1962) 163-288).

[21] S.L. LEE, B-splines for cardinal hermite interpolation, Linear Algebra and Applications 12, (1975) 269-280.

[22] A.V. Oppenheim and R.W. Schafer, Discrete-Time Signal Processing, Prentice Hall Signal Processing Series. Prentice Hall, Englewood Cliffs, NJ, 1989.

[23] F. Riesz AND B. Sz.-NaGY, Functional Analysis, Frederick Ungar, New York, 1955.

[24] S. Roch AND B. SilbermanN, $C^{*}$-algebra techniques in numerical analysis, J. Operator Theory 35, (1996) 241-280. 
[25] L. Rodman, An Introduction to Operator Polynomials, volume 38 of Operator Theory: Advances and Applications, Birkhäuser, Basel-Boston, 1989.

[26] I.J. Schoenberg, Cardinal Spline Interpolation, volume 12 of CBMS-NSF Regional Conference Series in Applied Mathematics, SIAM, Philadelphia, PA, 1973.

[27] G. Strang and T. Nguyen, Wavelets and Filter Banks, Wellesley-Cambridge Press, Wellesley, MA, 1996.

[28] G. WILSON, Factorization of the covariance generating function of a pure moving average process, SIAM J. Numer. Anal. 6(1), (1969) 1-7. 